UNITED STATES DEPARTMENT OF THE INTERIOR GEOLOGICAL SURVEY

GEOHYDROLOGIC DATA FOR TEST WELL UE-25b\#I

NEVADA TEST SITE, NYE COUNTY, NEVADA

by

D. H. Lobmeyer, M. S. Whitfield, Jr., R. X. Lahoud, and Laura Bruckheimer

Open-File Report 83-855

Prepared in cooperation with the

Nevada Operations office

U.S. Department of Enargy

(Interagency Agreement DE-AI08-78ET44802)

Denver, Colorado

1983 


\section{UNITED STATES DEPARTMENT' OF THE INTERIOR \\ WILIIAM P. CLARK, Secretary \\ GEOLOGICAL SURVEY \\ Dallas L. Peck, Director}

For additional information write to:

Chief, Nuclear Hydroiogy Program Water Resources Division U.S. Geological Survey Box 25046, Mail Stop 416 Denver Federal Center Lakewood, co 80225
Copies of this report can be purchased from:

Open-File Services Section Western Distribution Branch U.S. Geological Survey Box 25425, Foderal Center Lakewood, Co 80225 [Telephone (303) 234-5888] 
Abstract-1

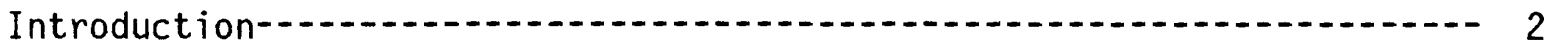

Drilling operations-an 2

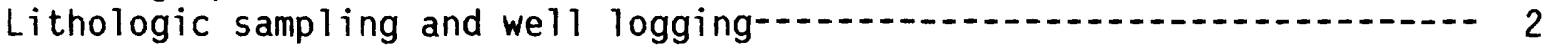

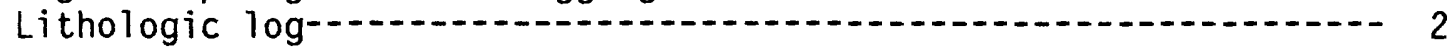

Hydrologic properties of core samples-18

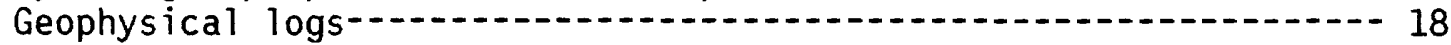

Hydrologic testing and water sampling

Water levels-a

Borehole-flow survey-a.t. 24

Pumping tests-(a)

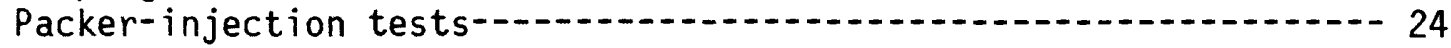

Chemical analyses of water-and 46

Selected references

\section{ILLUSTRATIONS}

Figure 1. Map showing location of test well UE-25b\#1 in southern

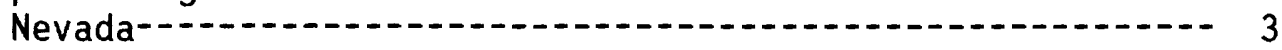

2. Map showing geographic features in the vicinity of test well UE-25b\#1--...-. 4

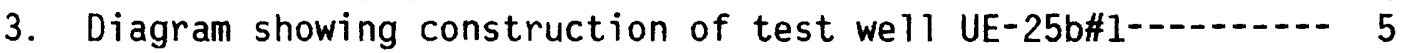
4-6. Graphs showing:

4. Drilling time for test well UE-25b\#1--...- 6

5. Generalized distribution of welding and induration of tuffs penetrated by test well UE-25b\#1-..........- 17

6. Porosity as determined by mechanical testing of

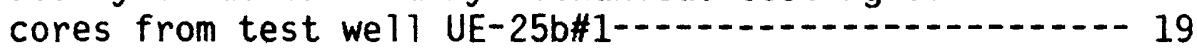

7. Diagram showing shear fractures as determined from cores, test well UE-25b\#1-- 21

8-13. Graphs showing:

8. Borehole-flow survey in test well UE-25b\#1, showing percent of pumping rate produced by intervals

9. Drawdown in test well UE-25b\#1 during pumping test $1---26$

10. Water-level recovery, pumping test 1 , test well UE-25b\#1--......-...- 27

11. Drawdown in test we 11 UE-25b\#1 and observation well UE-25a\#1 during pumping test 2--...... 28

12. Water-level recovery, pumping test 2 , test well UE-25b\#1 and observation well UE-25a\#1-............- 29

13. Drawdown in test well UE-25b\#1 and observation well UE-25a\#1 during pumping test 3-... 30 
Figures 14-28. Graphs showing packer-injection test for test well

$$
\text { UE-25b\#1: }
$$

14. Interval from 514 to 579 meters.................. 31

15. Interval from 505 to 579 meters.................. 32

16. Interval from 477 to 579 meters................. 33

17. Interval from 491 to 504 meters

18. Interval from 477 to 491 meters

19. Interval from 1,006 to 1,220 meters $\ldots \ldots \ldots$

20. Interval from 792 to 1,220 meters $\ldots \ldots \ldots \ldots$

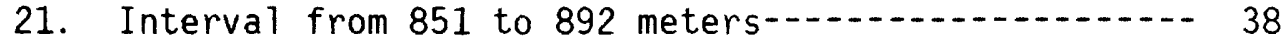

22. Interval from 820 to 860 meters

23. Interval from 779 to 820 meters

24. Interval from 743 to 783 meters

25. Interval from 703 to 743 meters-... 42

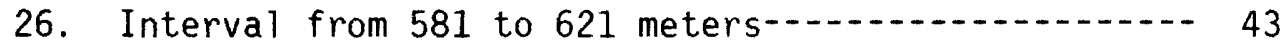

27. Interval from 518 to 545 meters.................. 44

28. Interval from 621 to 661 meters

\section{TABLES}

Table 1. Lithologic log for test well UE-25b\#1_... 7

2. Results of laboratory analyses for hydrologic properties of core samples from test well UE-25b\#1-..... 20

3. Summary of geophysical well logs made in test well UE-25b\#1--. 22

4. Summary of hydrologic tests in test well UE-?5b\#1-........ 23

5. Results of chemical analyses of water samples from test well

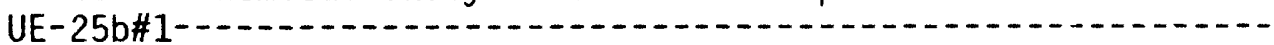




\section{CONVERSION TABLE}

For those readers who prefer to use inch-pound rather than metric units, conversion factors for the terms used in this report are listed below:

Metric unit

millimeter $(\mathrm{mm})$

gram per cubic centimeter

kilometer

meter (m)

degree Celsius

meter per day

milligram per liter

liter per second (L/s)

liter (L)
Multiply by

$$
\begin{aligned}
& 3.937 \times 10^{-2} \\
& 62.43 \\
& 6.214 \times 10^{-1} \\
& 3.281 \\
& 1.8^{\circ} \mathrm{C}+32 \\
& 3.281 \\
& 11.0 \\
& 1.585 \times 10^{1} 1 \\
& 2.642 \times 10^{-1}
\end{aligned}
$$

To obtain inch-pound unit

inch

pound per cubic foot

mile

foot

degree Fahrenheit

foot per day

part per million

gallon per minute

gallon

\section{${ }^{1}$ Approximate.}

National Geodetic Vertical Datum of 1929 (NGVD)--a geodetic datum derived from a general adjustment of the first-order level nets of both the United States and Canada, formerly called Mean Sea Level; it will be referred to as sea level in this report. 


\title{
GEOHYDROLOGIC DATA FOR TEST WELL UE-25b\#1, NEVADA TEST SITE, NYE COUNTY, NEVADA
}

By D. H. Lobmeyer, M. S. Whitfield, Jr., R. G. Lahoud, and Laura Bruckheimer

\begin{abstract}
This report presents data collected to determine the hydraulic characteristics of rocks penetrated in test well UE-25b\#1. This well is one of a series of test wells drilled in and near the southwestern part of the Nevada Test Site in a program conducted in cooperation with the U.S. Department of Energy. These investigations are part of the Nevada Nuclear Waste Storage Investigations to identify suitable sites for storage of high-level radioactive wastes. Data on drilling operations, lithology, core analyses, borehole geophysics, hydrologic monitoring, hydraulic testing, and ground-water chemistry for test well UE-25b\#1 are contained in this report.
\end{abstract}




\section{INTRODUCTION}

The U.S. Geological Survey has been conducting investigations at Yucca Mountain, Nevada, to evaluate the hydrologic and geologic suitability of this site for storing high-level nuclear waste in an underground mined repository. The investigations are part of the Nevada Nuclear Waste Storage Investigations being conducted in cooperation with the U.S. Department of Energy, Nevada Operations office. Test drilling has been a principal method of investigation. This report presents geohydrologic and drill-hole data from hydrologic test well UE-25b\#1. All data tables presented were compiled by the authors except where otherwise noted.

Test we 11 UE-25b\#1 is located in Nye County, Nevada, approximately $145 \mathrm{~km}$ northwest of Las Vegas on the Nevada Test Site (fig. 1). Coordinates of the well site are Nevada Coordinate system, Central Zone N 765,244 and E 566,417. The well is situated in a major wash, which trends northwest from Fortymile Wash on the east flank of Yucca Mountain (fig. 2). The altitude of the well is $1,200.3 \mathrm{~m}$ above sea level. A previously drilled nearby core hole, UE-25a\#1 (fig. 2), was used as an observation well during the aquifer tests. This well is $107 \mathrm{~m}$ south-southwest of UE-25b\#1 in the wash at an altitude of $1,199 \mathrm{~m}$.

\section{DRILLING OPERATIONS}

Drilling of test well UE-25b\#1 was started April 3, 1981, and completed July 31 , 1981, to a total depth of $1,220 \mathrm{~m}$. Drilling was performed by rotary drill with air-foam as the circulating medium. A hole $222 \mathrm{~mm}$ in diameter was originally drilled to $579 \mathrm{~m}$. The first episode of testing was conducted at this depth. The hole was subsequently enlarged, cased and deepened. Construction data for the completed test well are shown in figure 3 .

The second episode of testing was performed shortly after completion to a total depth of $1,220 \mathrm{~m}$. A third testing episode, using packers to determine the vertical head for the four most productive zones, was conducted June 8 and 9 , 1982. A graphic summary of the time required to drill this well is given in figure 4 .

\section{LITHOLOGIC SAMPLING AND WELL LOGGING}

\section{Lithologic Log}

The rocks penetrated in test well UE-25b\#1 are of volcanic and volcanicclastic origin. Lithologic descriptions from drill cuttings and cores are given in table 1. Ash-flow tuffs are the predominant rock type in this section. Thin beds of air-fall reworked bedded tuffs separate many of the ash-flow units. The tuffs have various degrees of welding and induration (fig. 5). 


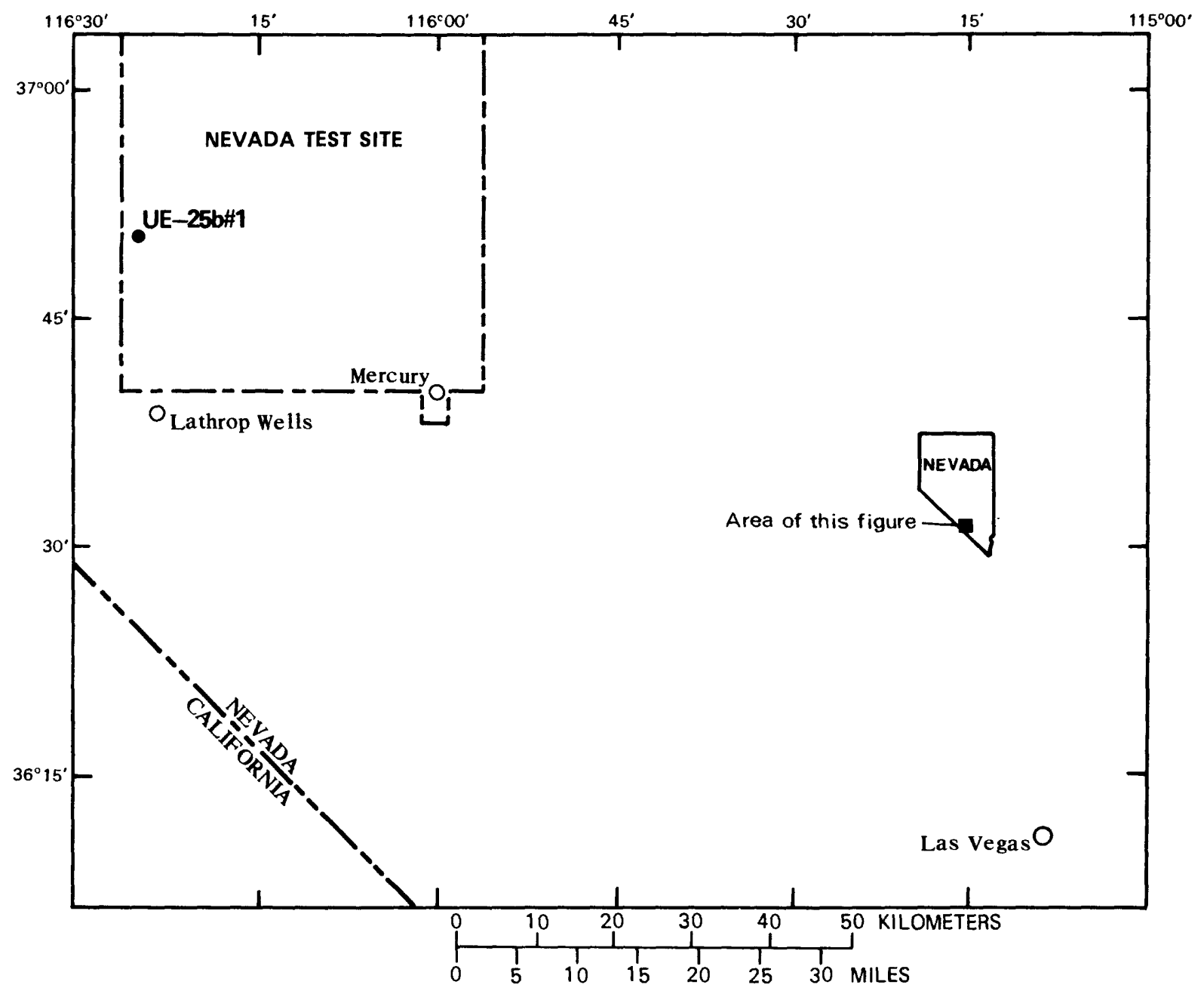

Figure 1.--Location of test well UE-25b\#1 in southern Nevada. 


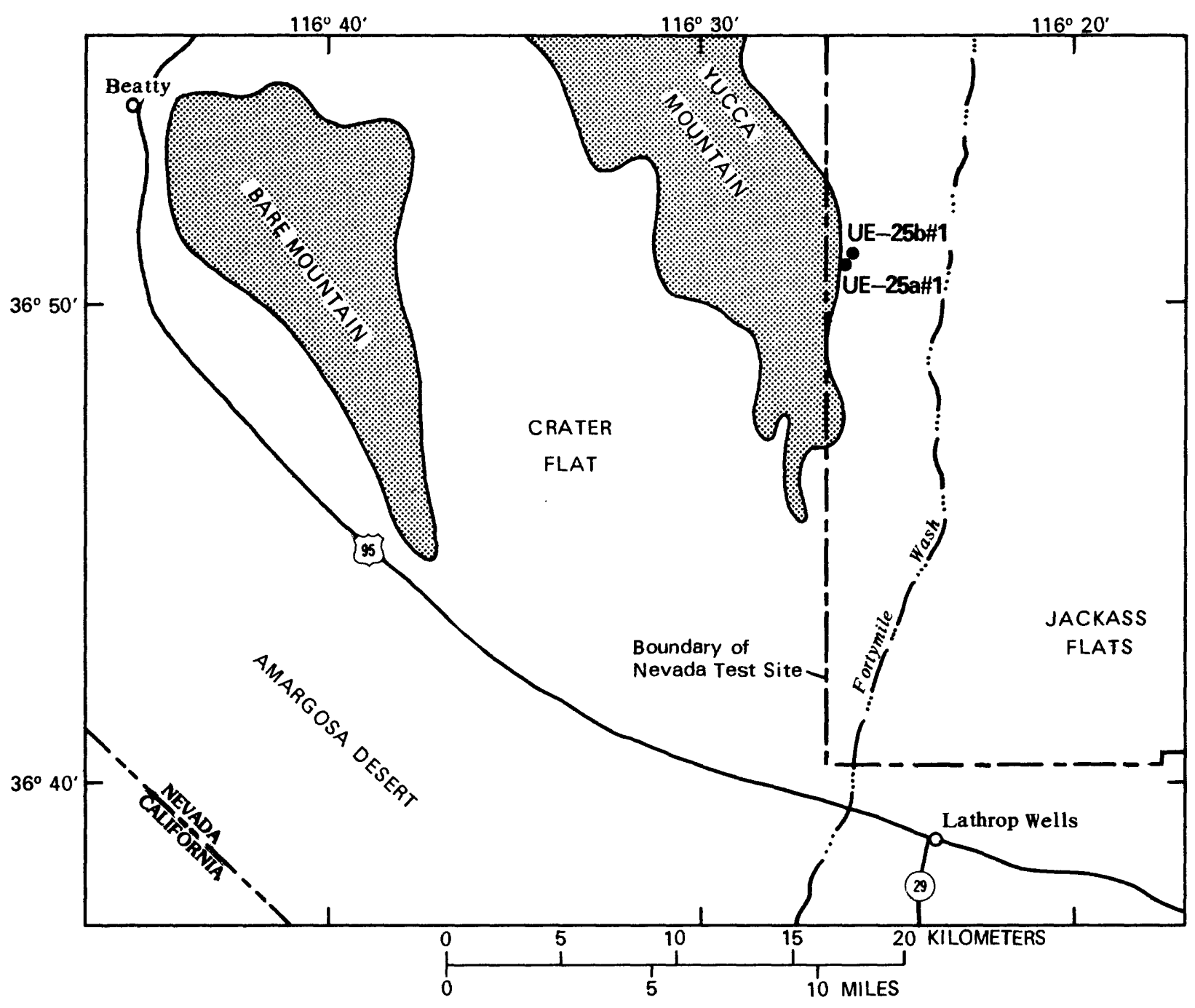

Figure 2.--Geographic features in the vicinity of test well UE-25b\#1. 


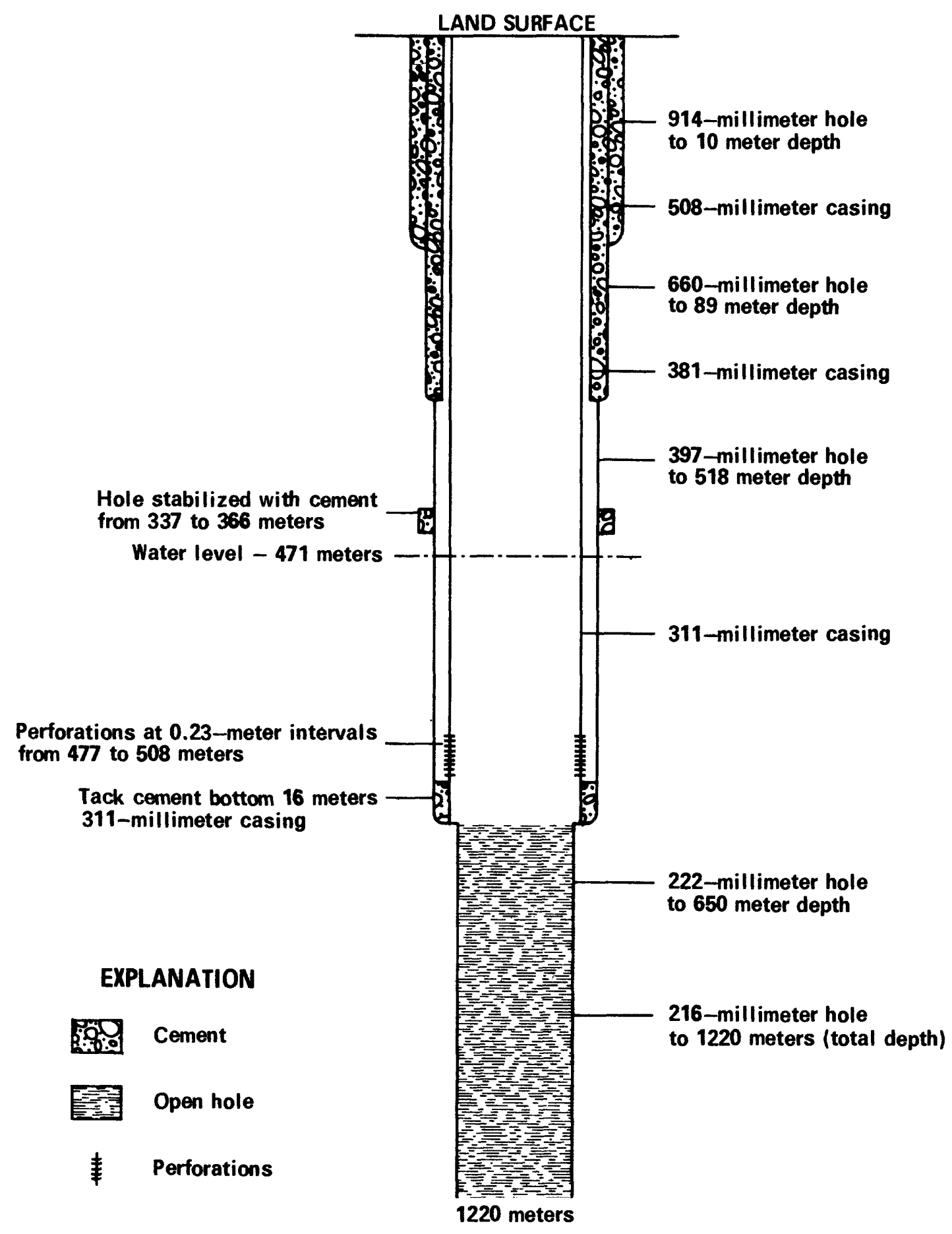

(DIAGRAM NOT TO SCALE)

Figure 3.--Construction of test well UE-25b\#1. 


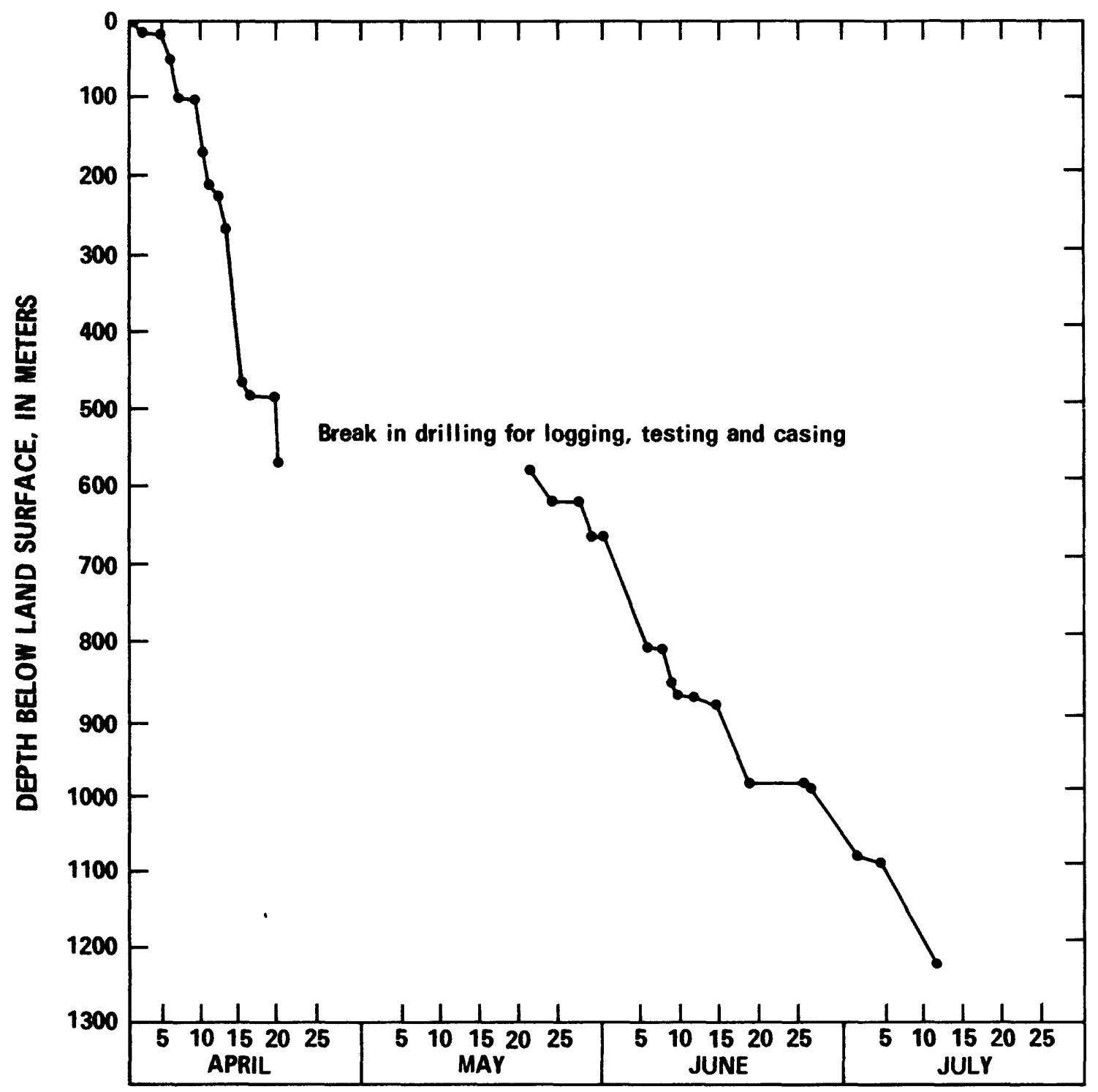

Figure 4.--Drilling time for test well UE-25b\#1. 
Table 1.--Lithologic log for test well UE-25b\#1

[Color designations are from the Rock Color Chart (Goddard and others, 1948); modified from R. W. Spengler, U.S. Geological Survey, written commun., 1983, and oral commun., 1983]

\begin{tabular}{lcc}
\hline Stratigraphy and 7ithologic description & $\begin{array}{c}\text { Thickness } \\
\text { of }\end{array}$ & $\begin{array}{c}\text { Depth to } \\
\text { bottom of }\end{array}$ \\
interval & interval \\
(meters) & (meters) \\
\hline
\end{tabular}

Alluvium

Grave 1, composed of fragments of Tiva Canyon and Yucca Mountain Members of Paintbrush Tuff, subangular to subrounded, few fragments coated with caliche.

45.7

45.7

Paintbrush Tuff

Tiva Canyon Member

Tuff, ash-flow, grayish-red, densely welded; grayish-red pumice; 1-2 percent phenocrysts.

18.3

64.0

Tuff, ash-flow, light-brown, partially to nonwelded; pale-yellowish-brown pumice, vitric, less than 1 percent phenocrysts.

9.1

73.1

Pah Canyon Member

Tuff, ash-flow, moderate-reddish-orange, palereddish-brown, nonwelded, vitric, grayish-orange pumice, vitric, less than 1 percent phenocrysts.

6.1

79.2

Bedded tuff (unnamed)

Tuff, bedded/reworked, grayish-yellow, vitric and devitrified, rich in pumice.

4.6

83.8

Topopah Spring Member

Tuff, ash-flow, grayish-red, densely welded (vitrophyre).

Tuff, ash-flow, grayish-red, densely welded, devitrified; blackish-red pumice, devitrified; 10 percent phenocrysts (quartz, sanidine, plagioclase, biotite, pyroxene) (quartz latitic caprock). 3.1 
Table 1.--Lithologic log for test well UE-25b\#1--Continued

\begin{tabular}{|c|c|c|}
\hline Stratigraphy and lithologic description & $\begin{array}{c}\text { Thickness } \\
\text { of } \\
\text { interval } \\
\text { (meters) }\end{array}$ & $\begin{array}{l}\text { Depth to } \\
\text { bottom of } \\
\text { interval } \\
\text { (meters) }\end{array}$ \\
\hline
\end{tabular}

Paintbrush Tuff--Continued

Topopah Spring Member--Continued

Tuff, ash-flow, grayish-red, light-brown, densely welded, devitrified; moderate-reddish-orange pumice, devitrified; 2-3 percent phenocrysts; Core samples collected from 164.6 to $169.5,170.7$ to $178.0,178.0$ to $182.6,222.5$ to $224.6,224.6$ to 225.6 , and 225.6 to $227.1 \mathrm{~m}$; samples collected from 170.7 to $178.0 \mathrm{~m}$ occur in a lithophysal zone. 306.3

394.7

Tuff, ash-flow, black, densely welded, vitric (vitrophyre).

10.7

405.4

Tuff, ash-flow, light-brown, moderately to partially welded, vitric; moderate-brown pumice, vitric; abundant black glass shards.

Bedded tuff (unnamed)

Tuff, bedded/reworked, dark-yellowish-orange, altered, abundant medium-dark-gray rhyolite lithic fragments.

10.7

422.2

Rhyolite lava and tuffs of Calico Hills (undivided)

Tuffaceous beds of Calico Hills (informal usage)

Tuff, ash-flow, light-brown, grayish-orange, yellowishorange, and yellowish-gray, nonwelded, zeolitized;

pumice, grayish-yellow and yellowish-gray, zeolitized; commoniy $1-3 \mathrm{~cm} ; 1-3$ percent phenocrysts; about 5 percent brownish-gray and medium-gray volcanic lithic fragments, rhyolitic; commonly 1-4 cm (core collected from 478.5 to $484.9 \mathrm{~m}$, recovered $6.4 \mathrm{~m}$ ).

138.7

560.8

Tuff, bedded, ash-fall(?), identified on density log and downhole television camera.

8.8

569.7

Crater Flat Tuff

Prow Pass Member

Tuff, ash-flow, moderate-reddish-orange, nonwelded to partially welded, zeolitized. 
Table 1.--Lithologic log for test well UE-25b\#1--Continued

\begin{tabular}{lcl}
\hline Stratigraphy and lithologic description & $\begin{array}{c}\text { Thickness } \\
\text { of } \\
\text { interval } \\
\text { (meters) }\end{array}$ & $\begin{array}{l}\text { Depth to } \\
\text { bottom of } \\
\text { interval } \\
\text { (meters) }\end{array}$ \\
\hline
\end{tabular}

Crater Flat Tuff--Continued
Prow Pass Member--Continued

Tuff, ash-flow, light-brownish-gray, partially

welded, devitrified; pumice, very light gray to

medium-dark-gray, 5-40 mm, devitrified; 10 percent

phenocrysts (quartz, sanidine, plagioclase,

biotite); 1-2 percent grayish-red mudstone and

dark-gray rhyolitic lithic fragments, commonly

less than $2 \mathrm{~cm}$.

$2.6 \quad 580.5$

Tuff, ash-flow, light-brown, partially welded, devitrified; light-gray, light-brownish-gray pumice, 5-40 $\mathrm{mm}$, devitrified; 10 percent phenocrysts (quartz, sanidine, plagioclase, biotite); less than 2 percent moderate-red mudstone lithic fragments, commonly less than $1 \mathrm{~cm}$.

$2.3 \quad 582.8$

Tuff, ash-flow, light-gray, medium-light-gray, lightbrownish-gray, light-olive-gray, partially welded; pumice, commonly white, very light gray, pinkish-gray, with vapor phase crystallization and devitrified, ranges from 2 to $60 \mathrm{~mm}$; 5 to 10 percent phenocrysts (quartz, plagioclase, sanidine, biotite); sparse moderate-red and brownish-gray mudstone and rhyolitic lithic fragments, commonly less than $1 \mathrm{~cm}$.

$18.2 \quad 601.0$

Tuff, ash-flow, light-red and light-brownish-gray (mottled), partially to moderately welded, devitrified; pumice pale-yellowish-orange, light-red, light-gray, devitrified, 2-40 mm; 5-10 percent phenocrysts (quartz, sanidine, plagioclase, biotite); sparse moderate-red and moderate-brown mudstone lithic fragments, 3-10 $\mathrm{mm}$; interval indicates an increase in near-vertical, iron-stained fractures.

$4.6 \quad 605.6$

Tuff, ash-flow, light-red, moderate-red, and pale-reddishbrown (mottled in a few places), grades from moderate to partial welding (moderately welded from 605.6 to about $614.0 \mathrm{~m}$ ), devitrified; pumice, moderate-orange-pink to moderate-red, devitrified, commonly ranges from 2 to $20 \mathrm{~mm}$, as large as $50 \mathrm{~mm}$; 5-10 percent phenocrysts, (quartz, sanidine, plagioclase, biotite, sparse orthopyroxene(?) pseudomorphs); 2-3 percent moderate-red and grayish-red mudstone lithic fragments, 5-30 $\mathrm{mm}$ in size; interval contains several prominent fractures at 612.3 , 
Table 1.--Lithologic log for test well UE-25b\#1--Continued

\begin{tabular}{|c|c|c|}
\hline Stratigraphy and lithologic description & $\begin{array}{l}\text { Thickness } \\
\text { of } \\
\text { interval } \\
\text { (meters) }\end{array}$ & $\begin{array}{l}\text { Depth to } \\
\text { bottom of } \\
\text { interval } \\
\text { (meters) }\end{array}$ \\
\hline
\end{tabular}

Crater Flat Tuff--Continued

Prow Pass Member--Continued

$613.7,617.5$, and $618.0 \mathrm{~m}$, fractures are commonly high angle and iron stained, some coated with $\mathrm{MnO}_{2}$; staining extends $1-2 \mathrm{~cm}$ outward from fracture face.

$17.6 \quad 623.2$

Tuff, ash-flow, moderate-red, light-red, and yellowishgray (upper $2.1 \mathrm{~m}$ mottled), partially welded to nonwelded, devitrified (slightly argillic); pumice, yellowish-gray, light-red, and moderate pink, devitrified (zeolitized), 5-30 mm; 5-10 percent phenocrysts (quartz, sanidine, plagioclase, biotite, sparse orthopyroxene(?) pseudomorphs); 2-5 percent moderate-red mudstone lithic fragments, commonly 2-10 mm.

$8.0 \quad 631.2$

Tuff, ash-flow, moderate-pink and grayish-yellow (mottled), nonwelded, devitrified (some vapor phase crystallization), partially zeolitized; pumice, moderate-pink, grayish-yellow, devitrified (vapor phase crystallization), 5-30 mm; 5-10 percent phenocrysts (quartz, sanidine, plagioclase, rare biotite); sparse moderate-reddish-brown mudstone and grayishred rhyolitic lithic fragments, commoniy 5-20 mm; near-vertical fault, containing breccia extends from 632.2 to $633.0 \mathrm{~m}$; breccia contains vitrophyre fragments from 633.5 to $633.7 \mathrm{~m}$.

$7.3 \quad 638.5$

Tuff, ash-flow, moderate-pink, very pale orange, moderate-orange-pink, partially welded, devitrified; pumice, grayish-orange-pink, moderate-pink, light-red, and grayish-yellow, devitrified, commonly 2-30 mm; 5-10 percent phenocrysts (quartz, sanidine, plagioclase, rare biotite); 3-5 percent moderate-reddish-brown and grayish-brown rhyolite lithic fragments, lithics commonly 5-30 mm; fault breccia cuts core from 638.8 to $639.5 \mathrm{~m}$.

Tuff, ash-flow, light-brown, grayish-orange-pink, and light-red, partially welded, devitrified (slightly zeolitized and silicified); pumice, moderate-orangepink, grayish-orange, and grayish-yellow, commonly 5-30 $\mathrm{mm}$, devitrified; $10-15$ percent phenocrysts (quartz, sanidine, plagioclase, rare biotite); 1-3 percent moderate-reddish-brown and grayish-red mudstone lithic fragments (rare grayish-red rhyolitic lithics), 5-30 $\mathrm{mm}$. 
Table 1.--Lithologic log for test well UE-25b\#1--Continued

\begin{tabular}{lcl}
\hline Stratigraphy and lithologic description & $\begin{array}{c}\text { Thickness } \\
\text { of } \\
\text { interval } \\
\text { (meters) }\end{array}$ & $\begin{array}{l}\text { Depth to } \\
\text { bottom of } \\
\text { interval } \\
\text { (meters) }\end{array}$ \\
\hline
\end{tabular}

Crater Flat Tuff--Continued

Prow Pass Member--Continued

Tuff, ash-flow, pale-yellowish-brown, moderateyellow-brown, light-brown, pale-brown, moderatered, light-olive-gray, and dark-greenish-gray, partially to moderately welded, devitrified (partially to moderately silicified); pumice, grayish-yellow, greenish-gray, yellow-gray, paleo] ive, and medium-gray, some vitric and silicified, commonly 5-30 $\mathrm{mm}$; 5 percent phenocrysts (quartz, sanidine, plagioclase, rare biotite); rare moderate-reddish-brown mudstone lithic fragments.

16.1

666.8

Tuff, ash-flow, grayish-orange, partially to moderately welded, devitrified (slightly silicified); pumice, grayish-yellow, grayish-orange-pink, grayishorange, light-brown, devitrified, slightly silicified, commonly 5-20 mm; 10 percent phenocrysts [quartz, sanidine, plagioclase, rare biotite, rare orthopyroxene(?)]; 1-3 percent moderate-reddish-brown mudstone and brownish-gray rhyolitic lithic fragments, commonly 5-30 mm; upper and lower contacts gradational.

Tuff, ash-flow, pale-yellowish-brown, greenish-gray, dark-greenish-gray, light-olive-gray, partially to moderately welded, devitrified, moderately to highly silicified and zeolitized; pumice, grayish-orange, moderate-brown, moderate-ye $110 w-g r e e n$, dark-greenishgray, devitrified, silicified, zeolitized, 5-30 mm; 10 percent phenocrysts (quartz, sanidine, plagioclase, rare biotite); 1-2 percent moderate-reddish-brown, moderate-red rhyolite and mudstone 1ithic fragments, commonly 5-30 mm, as large as $60 \mathrm{~mm}$; lower contact gradational.

Tuff, ash-flow, pale-yellowish-brown, partially welded, devitrified (upper $4.9 \mathrm{~m}$ slightly silicified); pumice, pale-yellowish-orange, grayish-orange, grayish-yellow, dark-yellowish-orange, commoniy 5-20 $\mathrm{cm} ; 10$ percent phenocrysts (quartz, sanidine, plagioclase, rare biotite); 2-3 percent light-brown duskyred, moderate-reddish-brown, greenish-gray, lightbrownish-gray rhyolitic and mudstone lithic fragments (rhyolite is the dominant); lower contact gradational. 13.6 
Table 1.--Lithologic Iog for test well UE-25b\#1--Continued

\begin{tabular}{ll}
\hline Stratigraphy and lithologic description & $\begin{array}{c}\text { Thickness } \\
\text { of } \\
\text { interval to } \\
\text { (meters) }\end{array}$ \\
$\begin{array}{c}\text { interval } \\
\text { (meters) }\end{array}$
\end{tabular}

Crater Flat Tuff--Continued

Prow Pass Member--Continued

Tuff, ash-flow, light-olive-gray, mottled moderateorange-pink to pale-reddish-brown, nonwelded, devitrified, zeolitized; pumice, pale-greenishyellow, grayish-orange, moderate-orange-pink, yellowish-gray, and pale-yellowish-orange, devitrified, commonly $1-3 \mathrm{~cm} ; 10$ percent phenocrysts (quartz, sanidine, plagioclase, rare biotite); 1-3 percent pale-reddish-brown, moderate-reddish-brown and brownish-gray rhyolitic and mudstone lithic fragments, commonly 5-20 mm, as large as $50 \mathrm{~mm}$; lithics increase to 5-6 percent in lower $11.3 \mathrm{~m}$ of subunit; lowermost $2.1 \mathrm{~m}$ of core is stained moderate-red and contains large, rounded pumice fragments, many of which range from $2-6 \mathrm{~cm}$ in size.

24.6

718.0

Bedded tuff (unnamed)

Tuff, bedded/reworked, air-fall, light-olive-gray, light-brownish-gray, moderate-red, devitrified (some beds silicified and(or) zeolitized); beds range in thickness from 0.1 to $0.4 \mathrm{~m}$; both gradational and abrupt contacts present; bedding inclined from $5^{\circ}$ to $8^{\circ}$ relative to core axis, basal contact inclined $19^{\circ}$.

Bullfrog Member

Tuff, ash-flow, light-brown, partially welded, devitrified; pumice, 1 ight-brown and moderatered, commoniy 5-20 mm, devitrified; 10-15 percent phenocrysts (quartz, plagioclase, sanidine, biotite); rare moderate-reddish-brown and dark-gray mudstone and rhyolitic lithic fragments, commonly 5-20 mm.

Tuff, ash-flow, grayish-orange-pink, partially welded, vapor phase crystallization; pumice, palered, pale-yellowish-brown, light-brown, commonly 5-20 $\mathrm{mm}$, as large as $60 \mathrm{~mm}$, vapor phase; 10-15 percent phenocrysts (quartz, plagioclase, sanidine, biotite); rare light-brown and grayish-orange-pink rhyolitic lithic fragments; lower contact gradational. 
Table 1.--Lithologic log for test well UE-25b\#1--Continued

\begin{tabular}{lcc}
\hline Stratigraphy and 1ithologic description & $\begin{array}{c}\text { Thickness } \\
\text { of }\end{array}$ & $\begin{array}{c}\text { Depth to } \\
\text { bottom of } \\
\text { interval } \\
\text { (meters) }\end{array}$ \\
\hline
\end{tabular}

Crater Flat Tuff--Continued

Bullfrog Member--Continued

Tuff, ash-flow, light-gray and light-brownish-gray, partially welded, vapor phase crystallization; pumice, light-brownish-gray, and medium-light-gray, commonly 5-30 mm, vapor phase crystallization; 10-15 percent phenocrysts (quartz, sanidine, plagioclase, biotite); rare (less than 1 percent) brownish-gray rhyolitic and mudstone 1ithic fragments, commonly 5-15 mm; lower contact gradational.

Tuff, ash-fall(?), partially welded(?), light-gray to medium-gray; pumice flattened, subtle concentrations of phenocrysts, lower contact gradational.

Tuff, ash-flow, light-brown and pale-reddish-brown, moderately welded, devitrified; pumice, light-brownishgray, and brownish-gray, devitrified, commonly 5-20 mm; 10 percent phenocrysts (quartz, plagioclase, sanidine, biotite; rare (less than 2 percent) brownish-gray and moderate-red rhyolite and mudstone lithic fragments, commonly 5-15 mm; lower contact gradational.

Tuff, ash-flow, moderate-orange-pink, grayish-orange, grayish-orange-pink, light-brown (lower $0.8 \mathrm{~m} \mathrm{pale-}$ olive), partially welded to nonwelded, moderately zeolitic (slightly argillic); pumice, pale-reddishbrown, grayish-red, light-brown, moderate-yellowgreen, and light-olive, moderately zeolitic, slightly argillic; commonly 5-25 mm, as large as $60 \mathrm{~mm}$ near the top of subunit; 10 percent phenocrysts (quartz, plagioclase, sanidine, biotite, hornblende); rare (less than 2 percent) brownish-gray, pale-reddishbrown and light-gray rhyolitic lithic fragments.

\section{Bedded tuff (unnamed)}

Tuff, bedded/reworked, grayish-red, pale-olive, pale-yellowish-brown, pale-greenish-yellow, yellowishgray, moderately zeolitized; pumice, white, palegreenish-yellow, grayish-orange-pink, zeolitized; sparse lithics, commonly less than $5 \mathrm{~mm}$; thick bedded to massive; beds commonly 0.4 to $0.9 \mathrm{~m}$ thick; contacts commonly gradational.

$9.1 \quad 878.6$ 
Table 1.--Lithologic log for test well UE-25b\#1--Continued

\begin{tabular}{ll}
\hline Stratigraphy and lithologic description & $\begin{array}{c}\text { Thickness } \\
\text { of } \\
\text { interval both to } \\
\text { (meters) }\end{array}$ \\
\hline
\end{tabular}

Crater Flat Tuff--Continued

Tram Member

Tuff, ash-flow, light-brown, light-brownish-gray, grayish-orange-pink, nonwelded to partially welded, zeolitic; pumice, pale-yellowish-orange, moderateorange-pink, very pale orange, and grayish-red, zeolitized (some argillic), commonly 5-20 mm; 10-15 percent phenocrysts (quartz, plagioclase, sanidine, biotite); sparse moderate-reddish-brown, brownishgray and greenish-gray rhyolitic to intermediate lava lithic fragments, commonly 5-25 mm, as large as $55 \mathrm{~mm}$ (lower $0.8 \mathrm{~m}$ of subunit contains abundant (40-50 percent) rhyolitic and tuffaceous lithic fragments, commonly grayish-orange-pink, brownishgray and grayish-red, commonly $3-7 \mathrm{~cm}$ in length; clay content increases downward within subunit.

$19.2 \quad 897.8$

Tuff, ash-flow, very light gray, light-brownishgray, partially welded, devitrified; pumice, lightbrownish-gray, yellowish-gray, light-gray, and medium-light-gray, devitrified, commonly $2-30 \mathrm{~mm}$; 10 percent phenocrysts (quartz, sanidine, plagioclase, biotite); 2-3 percent dusky-red, brownish-gray, grayish-red, olive-gray, and light-gray rhyolitic to intermediate lava lithics and tuffaceous lithic fragments, commonly 5-20 mm, a few as large as $40 \mathrm{~mm}$.

Tuff, bedded (thinly laminated) and tuffaceous sandstone wel1-sorted, grayish-pink to moderate-red; laminations commonly 2-20 mm thick; laminations inclined $5^{\circ}$ relative to core axis.

Tuff, ash-flow, light-brownish-gray, partially welded, devitrified; pumice, light-gray and light-brownishgray, devitrified, commonly 5-20 mm; 10 percent phenocrysts (quartz, sanidine, plagioclase, biotite); 2-3 percent light-brown, pale-brown, brownish-gray rhyolitic to intermediate lava lithic fragments, commonly 4-20 mm.

Tuff, ash-flow, light-brown to pale-yellowish-brown, moderately welded, devitrified; pumice, grayishorange-pink, pale-yellowish-brown, light-brownishgray, devitrified, commonly $5-30 \mathrm{~mm}$; 10 percent phenocrysts (quartz, sanidine, plagioclase, biotite); 1-2 percent brownish-gray, moderate-brown, pale-brown 
Table 1.--Lithologic log for test well UE-25b\#1--Continued

\begin{tabular}{|c|c|c|}
\hline Stratigraphy and lithologic description & $\begin{array}{c}\text { Thickness } \\
\text { of } \\
\text { interval } \\
\text { (meters) }\end{array}$ & $\begin{array}{l}\text { Depth to } \\
\text { bottom of } \\
\text { interval } \\
\text { (meters) }\end{array}$ \\
\hline
\end{tabular}

Crater Flat Tuff--Continued

Tram Member--Continued

rhyolitic to intermediate lava and tuffaceous lithic fragments, commonly 5-30 mm; upper and lower contacts gradational.

5.7966 .5

Tuff, ash-flow, grayish-red, medium-dark-gray, darkgray, and pale-brown, moderately welded, devitrified (slightly to moderately silicified) pumice, grayish-red, pale-brown, and pale-yellow-brown, devitrified (some argilic), commonly 5-20 mm; 2-3 percent moderate-brown, brownish-gray, rhyolitic to intermediate lava and tuffaceous lithic fragments, commonly 5-20 mm, as large as $60 \mathrm{~mm}$; upper and lower contacts gradational.

21.6

988.1

Tuff, ash-flow, medium-light-gray to medium-dark-gray, moderately to partially welded, devitrified; pumice, very light gray, light-brownish-gray, grayish-yellow, moderate-pink pumice, argillic, commonly 5-20 mm; 10 percent phenocrysts (quartz, sanidine, plagioclase, biotite); 3-10 percent grayish-red, pale-brown, brownish-gray, and medium-dark-gray rhyolitic lava and tuffaceous lithic fragments, commonly $5-30 \mathrm{~mm}$, as large as $70 \mathrm{~mm}$; interval from 994.3 to $994.7 \mathrm{~m}$ contains 30-40 percent lithics.

$16.3 \quad 1,004.4$

Tuff, ash-flow, greenish-gray, light-brownish-gray, light-olive-gray, partially to moderately welded, devitrified (slightly zeolitized and argillic); pumice, light-gray, brownish-gray, light-brownish-gray and white, argillic and zeolitized, commonly 5-30 mm; 1015 percent phenocrysts (quartz, sanidine, plagioclase, biotite); 5-10 percent rhyolitic lava and tuffaceous lithic fragments, lithics commonly medium-dark-gray, brownish-gray, pale-brown, and medium-gray, commonly 5-30 mm; upper comtact gradational, lower contact sharp.

Tuff, ash-flow, yellowish-gray, light-greenish-gray, grayish-yellow-green, pale-yellowish-green, grayishgreen, light-olive, and greenish-gray, partially welded, argillic and zeolitized (has a bleached appearance); pumice, grayish-green, greenish-gray, and white, argillic and zeolitized, commonly $5-20 \mathrm{~mm} ; 10$ percent phenocrysts (quartz, sanidine, plagioclase, 
Table 1.--Lithologic log for test well UE-25b\#1--Continued

\begin{tabular}{|c|c|c|}
\hline Stratigraphy and lithologic description & $\begin{array}{c}\text { Thickness } \\
\text { of } \\
\text { interval } \\
\text { (meters) }\end{array}$ & $\begin{array}{l}\text { Depth to } \\
\text { bottom of } \\
\text { interval } \\
\text { (meters) }\end{array}$ \\
\hline
\end{tabular}

Crater Flat Tuff--Continued

Tram Member--Continued

biotite); rare (less than 1 percent) finely disseminated pyrite in matrix and lithic fragments; 10-25 percent light-brownish-gray, brownish-gray, light-olive-gray, medium-light-gray, dark-gray, and greenish-gray rhyolitic to intermediate lava and tuffaceous lithic fragments, commonly 5-20 mm, rarely larger than $40 \mathrm{~mm}$; clay-rich fault zone from $1,076.2$ to $1,077.2,1,078.0$ to $1,078.2$, and $1,080.5$ to $1,081.3 \mathrm{~m}$ (lowermost of the three zones is completely healed).

$164.8 \quad 1,189.0$

Bedded tuff (unnamed)

Tuff, bedded/reworked, dark-gray, ol ive-gray, yellowishgray, greenish-gray to dark-greenish-gray, moderately to highly indurated, zeolitic, most contacts gradaational, where sharp, contacts generally are inclined from $3^{\circ}$ to $5^{\circ}$ relative to the core axis; individual beds commonly range in thickness from 0.1 to $1.7 \mathrm{~m}$; most beds appear reworked and contain few large pumice fragments; where present pumice is white, yellowishgray, moderate-yellow, and green, argillic and zeolitized; pumice-rich intervals present from $1,189.4$ to $1,189.6,1,189.9$ to $1,191.6,1,193.0$ to $1,193.4$, and $1,206.5$ to $1,206.6 \mathrm{~m}$; lower contact gradational.

$18.1 \quad 1,207.1$

\section{Lithic Ridge Tuff}

Tuff, ash-flow, light-brown, olive-gray, light-olivegray, greenish-gray, moderately welded, devitrified (argillic and zeolitized); pumice, dark-greenish-gray, greenish-gray, and moderate-yellow-green, argillic and zeolitized, commonly 5-20 mm; 10 percent phenocrysts (quartz, sanidine, plagioclase, biotite, rare sphene); 5-10 percent dark-gray, dark-greenish-gray, and brownish-gray rhyolitic to intermediate lava lithic fragments, commonly $1-4 \mathrm{~cm}$.

$$
\text { Total depth }=12.8 \quad 1,219.9
$$




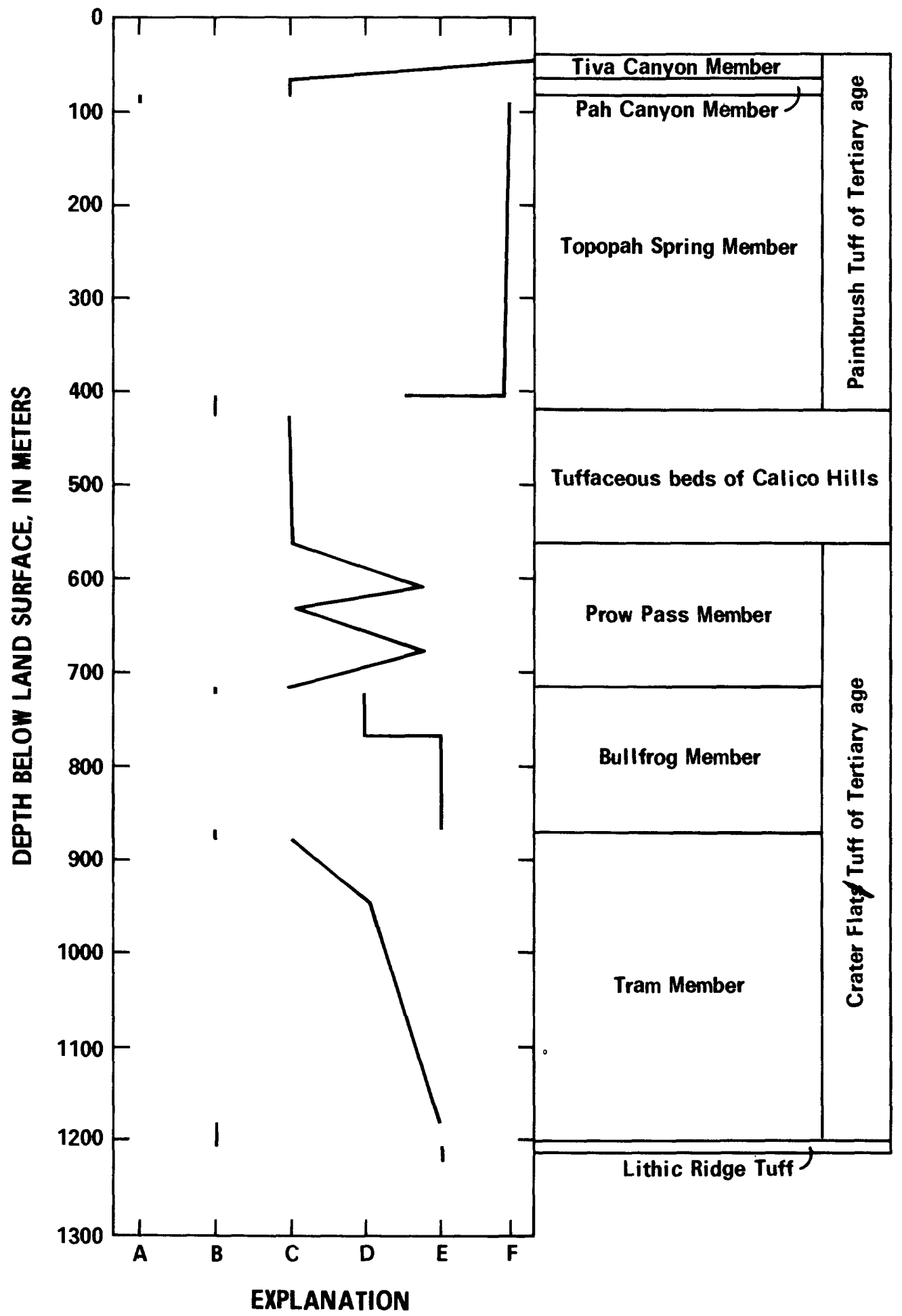

INDURATION: A, slightly; B, moderately

WELDING: C, nonwelded; $D$, partly

$E$, moderate; $F$, densely

Figure 5.--Generalized distribution of welding and induration of tuffs penetrated by test well UE-25b\#1. 


\section{Hydrologic Properties of Core Samples}

Sandia National Laboratories (contractor of U.S. Department of Energy) performed tests for selected physical properties on 127 core samples from the interval 589 to $1,186 \mathrm{~m}$. Distribution of matrix porosity is shown in figure 6. Physical property tests were performed by Holmes \& Narver Materials Testing Laboratory (contractor of U.S. Department of Energy) on 14 core samples, including one sample from the unsaturated zone (table 2).

A $\log$ of shear fractures was compiled from examination of the cores. Shear fractures are defined as fractures showing slickensides and are used in this report to define fault zones. Some prominent fault zones and numerous smaller fault zones were identified (fig. 7). Open fractures that occur extensively in a formation are related to very permeable zones.

\section{Geophysical Logs}

Geophysical logs of test well UE-25b\#1 were made for lithological definition; correlation with logs of nearby wells; obtaining data on porosity, fractures, and permeability; obtaining fluid levels; locating casing perforations and cement; and gaging the diameter of the well. Geophysical logs were used to help select hydraulic-test intervals. A summary of geophysical logs made in this well and the intervals that were logged are shown in table 3 .

Geophysical logs that can be used to identify water-yielding zones include the following: (1) Downhole televiewer, which shows zones with multiple low-angle fractures; (2) self-potential curve, which may change opposite water-yielding zones; and (3) temperature log where the gradient changes opposite water-yielding zones.

\section{HYDROLOGIC TESTING AND WATER SAMPLING}

The types of hydraulic tests performed in test well UE-25b\#1, intervals tested, duration of tests, and type of packer used are shown in table 4.

\section{Water Leve1s}

Water-level observations and measurements in test well UE-25b\#1 were made during drilling, during hydraulic testing, and after testing was completed. The purposes of these observations and measurements were: (1) To locate possible perched water in the unsaturated zones; (2) to determine depth at which water saturation occurs; and (3) to determine hydraulic heads in the well for specific zones. 


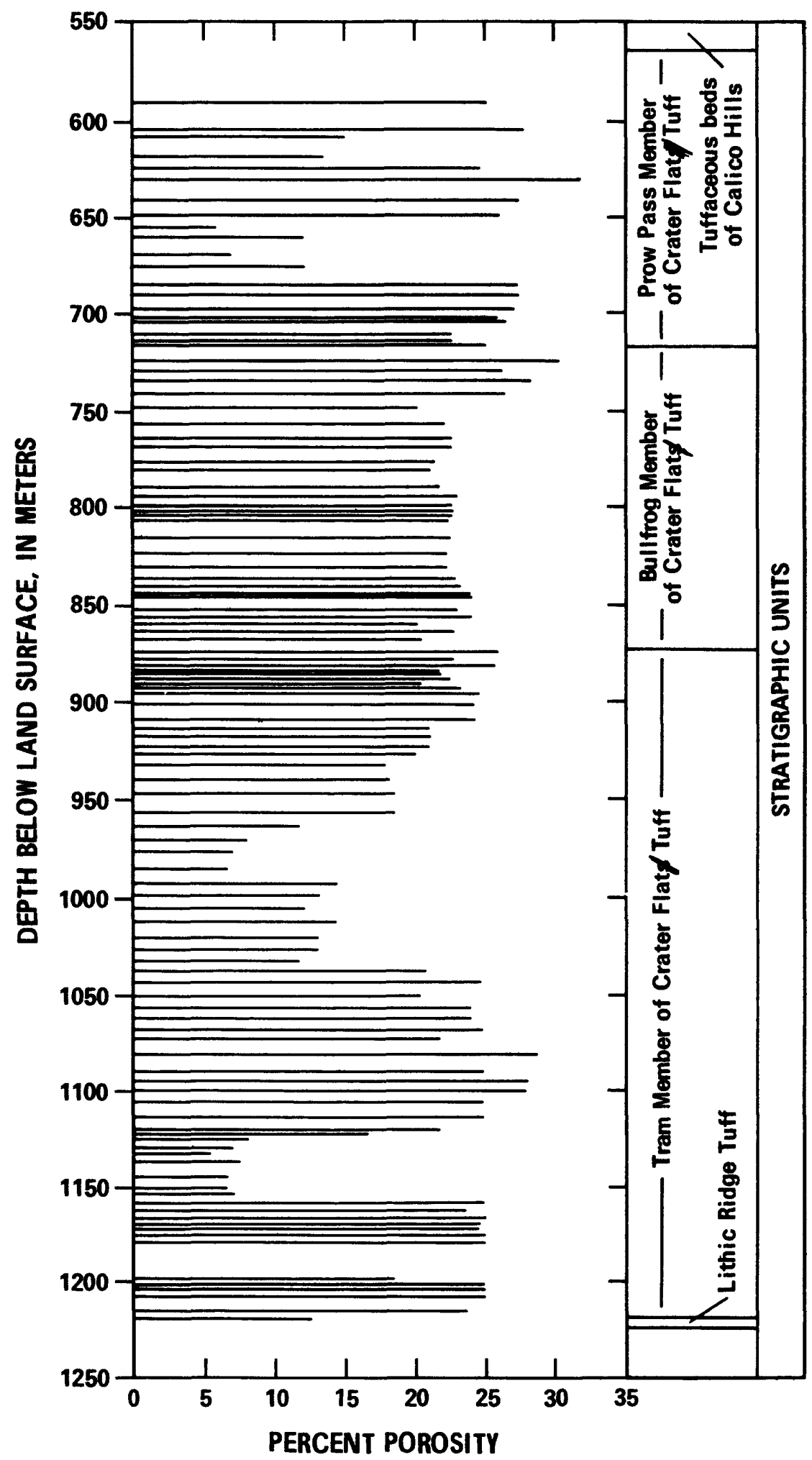

Figure 6.--Porosity as determined by mechanical testing of cores from test well UE-25b\#1. 


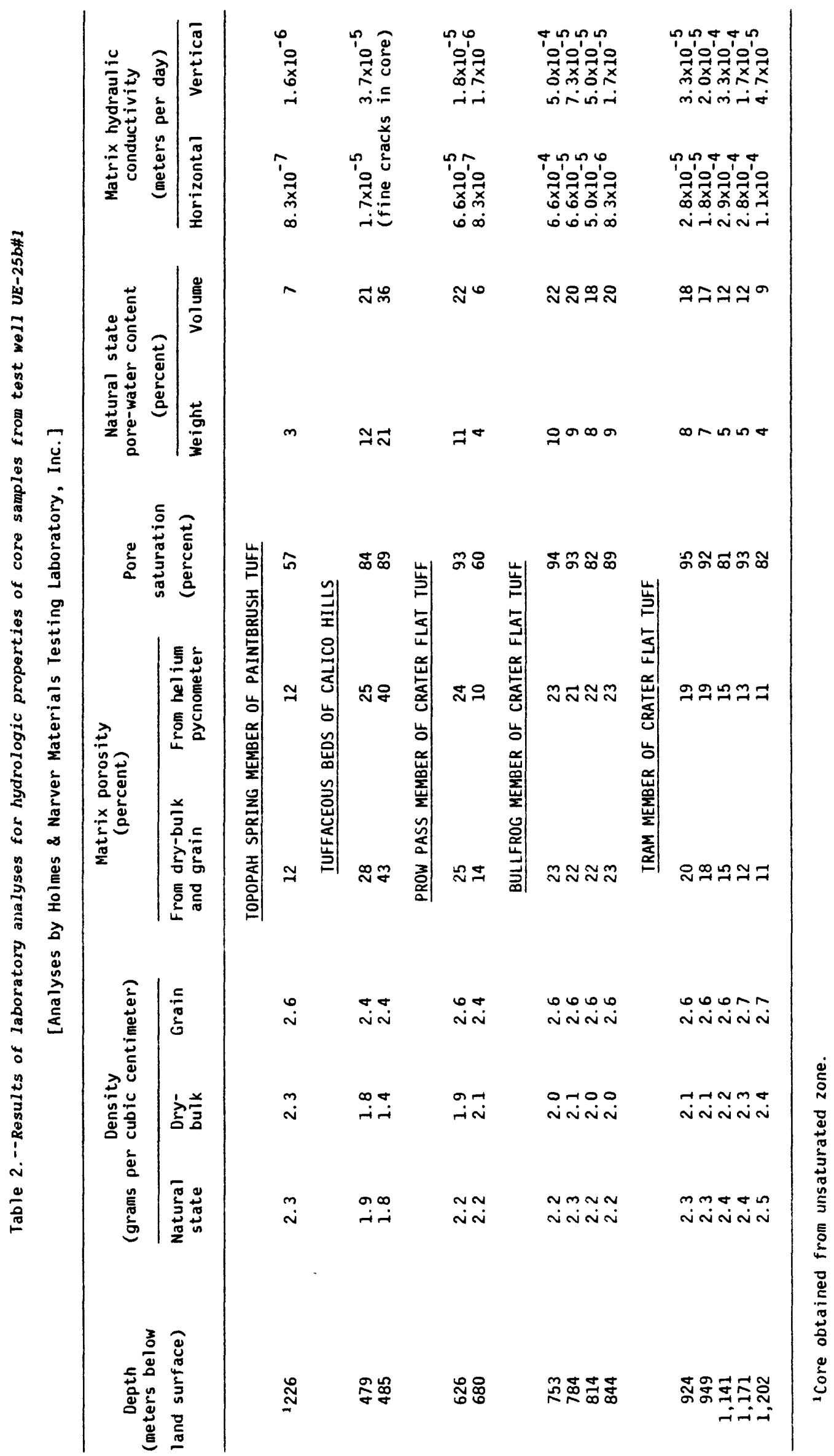




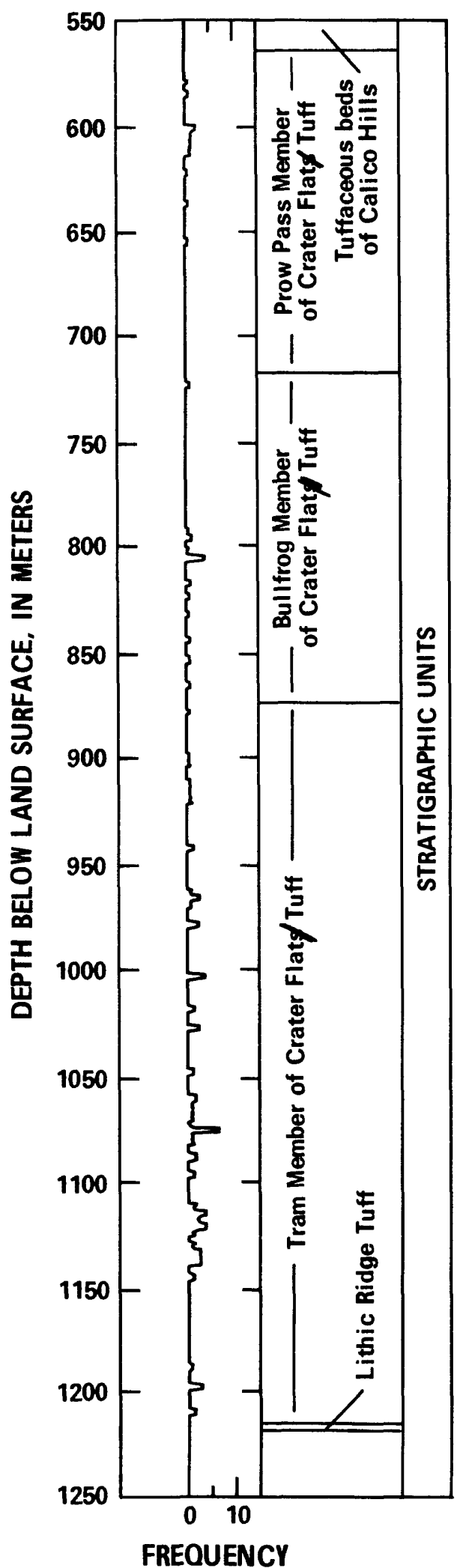

Figure 7.--Shear fractures as determined from cores, test well UE-25b\#1. 
Table 3.--Summary of geophysical well logs made in test well UE-25b\#1

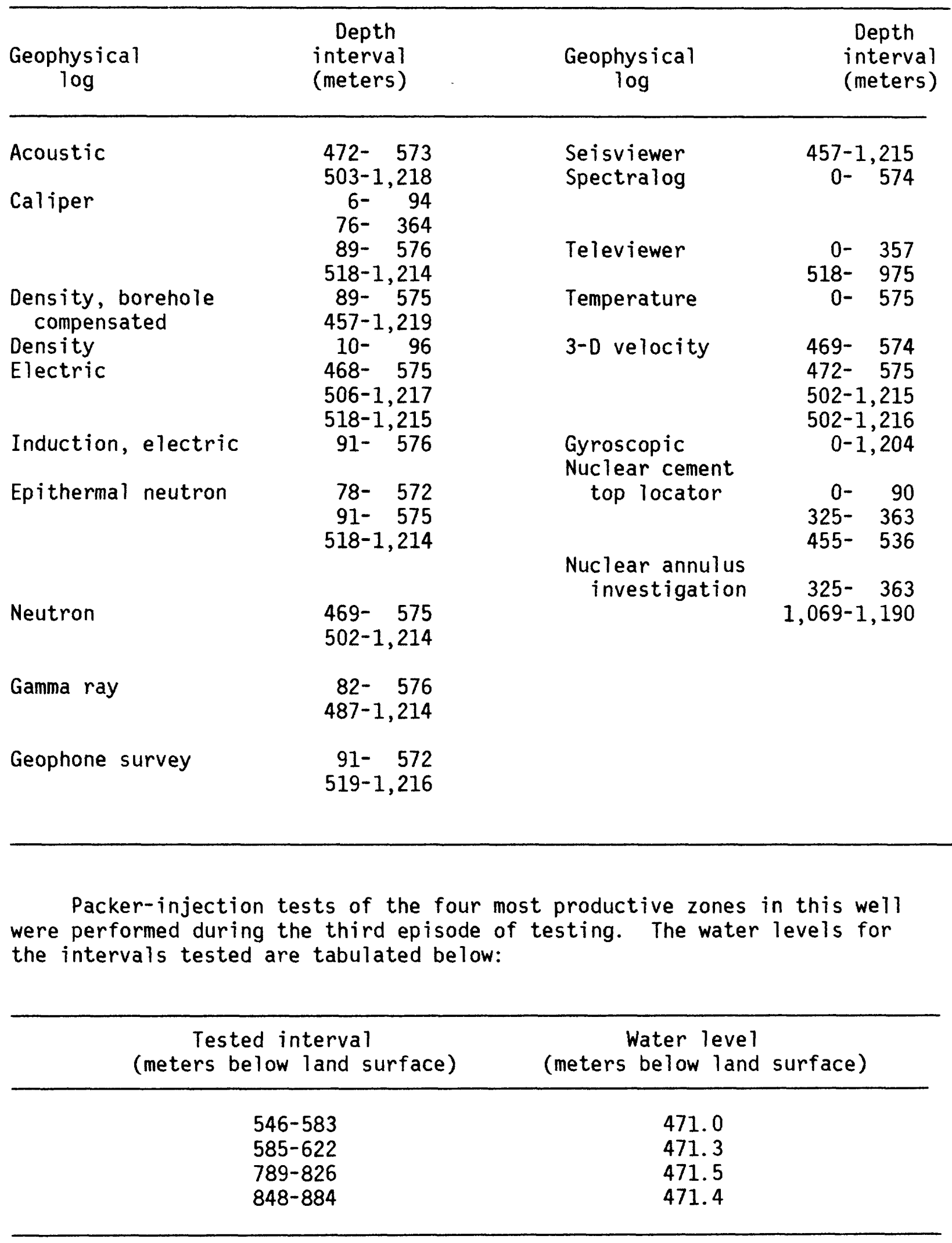



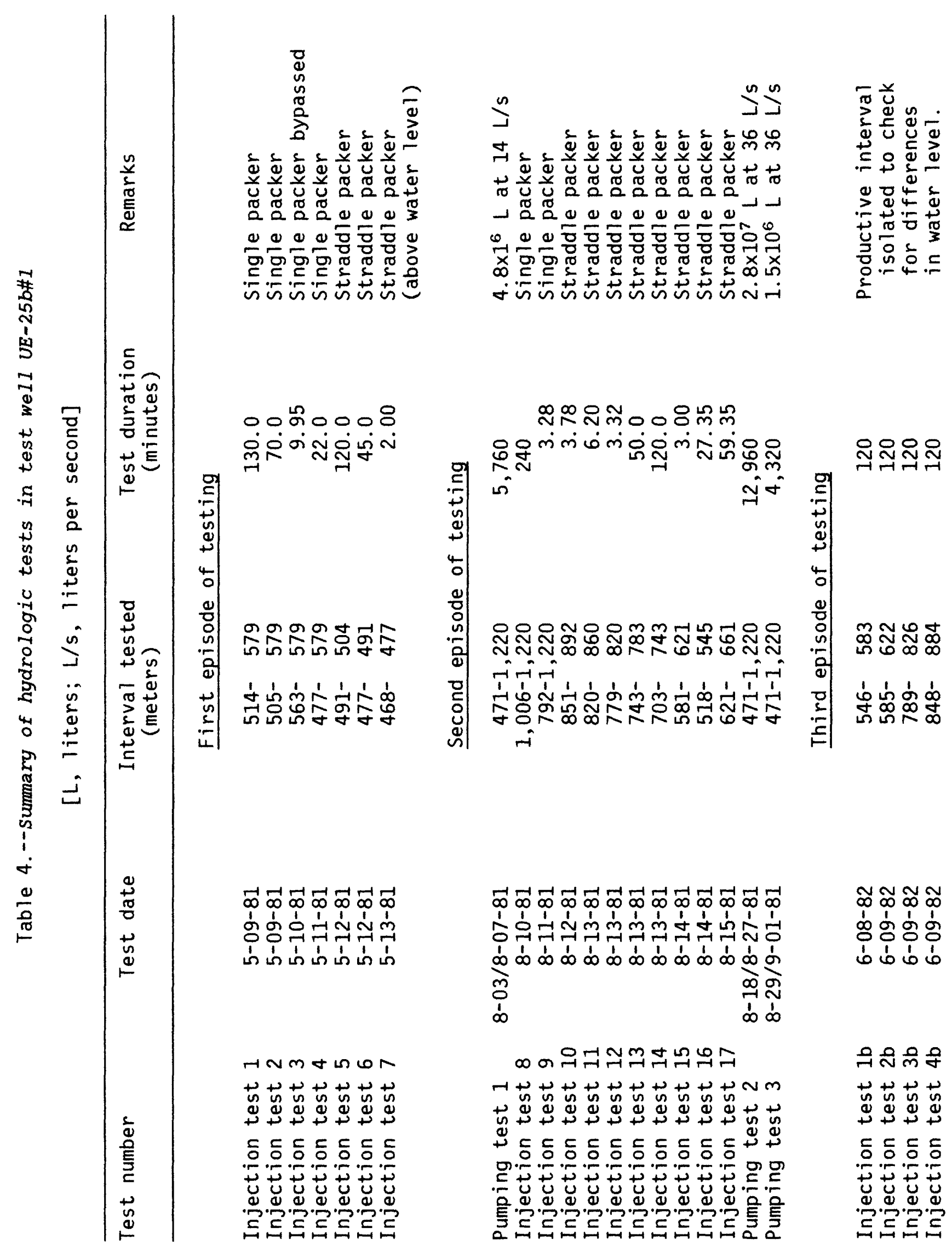

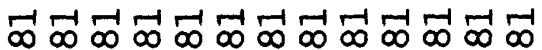

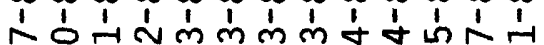

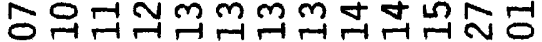

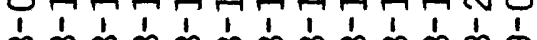

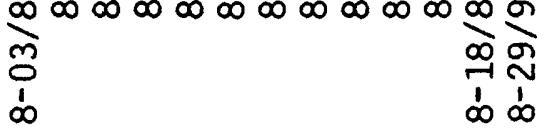

พิ $\approx \widetilde{\infty}$ 1 1 1 \%응용용 فأ

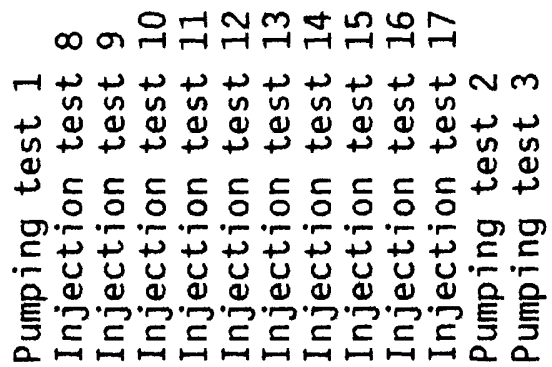

윽 슬ㅇㅇ

$++\omega$ (1) \&

등 등 등 등

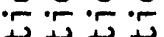
4 ن

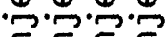

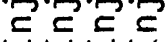




\section{Borehole-Flow Survey}

Borehole-flow surveys are used to measure vertical flow of water at selected intervals in the well while water is pumped into or out of the well. A slug of iodine-131 is released into the well at those intervals and is tracked past two gamma detectors to determine the velocity of the water. The velocity multiplied by the cross-sectional area determines the rate of flow in the well at a selected depth.

Two borehole-flow surveys were made in test well UE-25b\#1. The first was made when the well was at a depth of $579 \mathrm{~m}$. Technical problems in the well construction caused difficulty in obtaining useful data. The second survey was made in conjunction with pumping test 1 , after the bottom of the casing had been cemented, and the well was at total depth. A schematic diagram of the flow survey is shown in figure 8 .

\section{Pumping Tests}

Pumping tests were made in test well UE-25b\#1 after the well had been drilled to its total depth. The test interval was 471 to $1,220 \mathrm{~m}$. A pump with a capacity of $15 \mathrm{~L} / \mathrm{s}$ was used for the first pumping test; pumping rate was $13.4 \mathrm{~L} / \mathrm{s}$. The drawdown and recovery curves for this test are shown in figures 8 and 9, respectively. The second pumping test was made using a 1 arger pump to cause more stress on the aquifer. A pump with a rated capacity of $32 \mathrm{~L} / \mathrm{s}$ was used, pumping at rates ranging from 26.5 to $36.8 \mathrm{~L} / \mathrm{s}$. Drawdown for test 2 is shown in figure 10, and the recovery data for this test are shown in figure 11 .

The third pumping test was made using the same pump as the second test; pumping rate was $35.8 \mathrm{~L} / \mathrm{s}$, the same rate as the last 2,900 minutes of test 2 . Drawdown data for the third test are shown in figure 12 . Since adequate recovery data were obtained in tests 1 and 2, no recovery data were collected after test 3 . Water-level data for recovery tests are plotted at a different scale from that used for the drawdown plots. During tests 2 and 3 , we 11 UE-25a\#1 was used as an observation we 11.

\section{Packer-Injection Tests}

Packer-injection tests were performed at intervals where hole size and configuration allowed setting of inflatable packers. A slug of water was injected into the interval between two packers or between one packer and the bottom of the hole. The decline of hydraulic head with time was monitored in the isolated interval. Twenty-one tests were made on the well during three testing periods. Packer-injection test intervals and testing information are given in table 4 . The ratio of remaining hydraulic head to original head, plotted against time on semilogarithmic paper, is shown in figures 13 through 28. The type of water-level measuring device, the radius of the tested interval $\left(R_{s}\right)$, and the hydraulic head above the water level $(H)$ also are shown. The radius of the tubing in which the water level was measured was $0.031 \mathrm{~m}$ for all tests. 


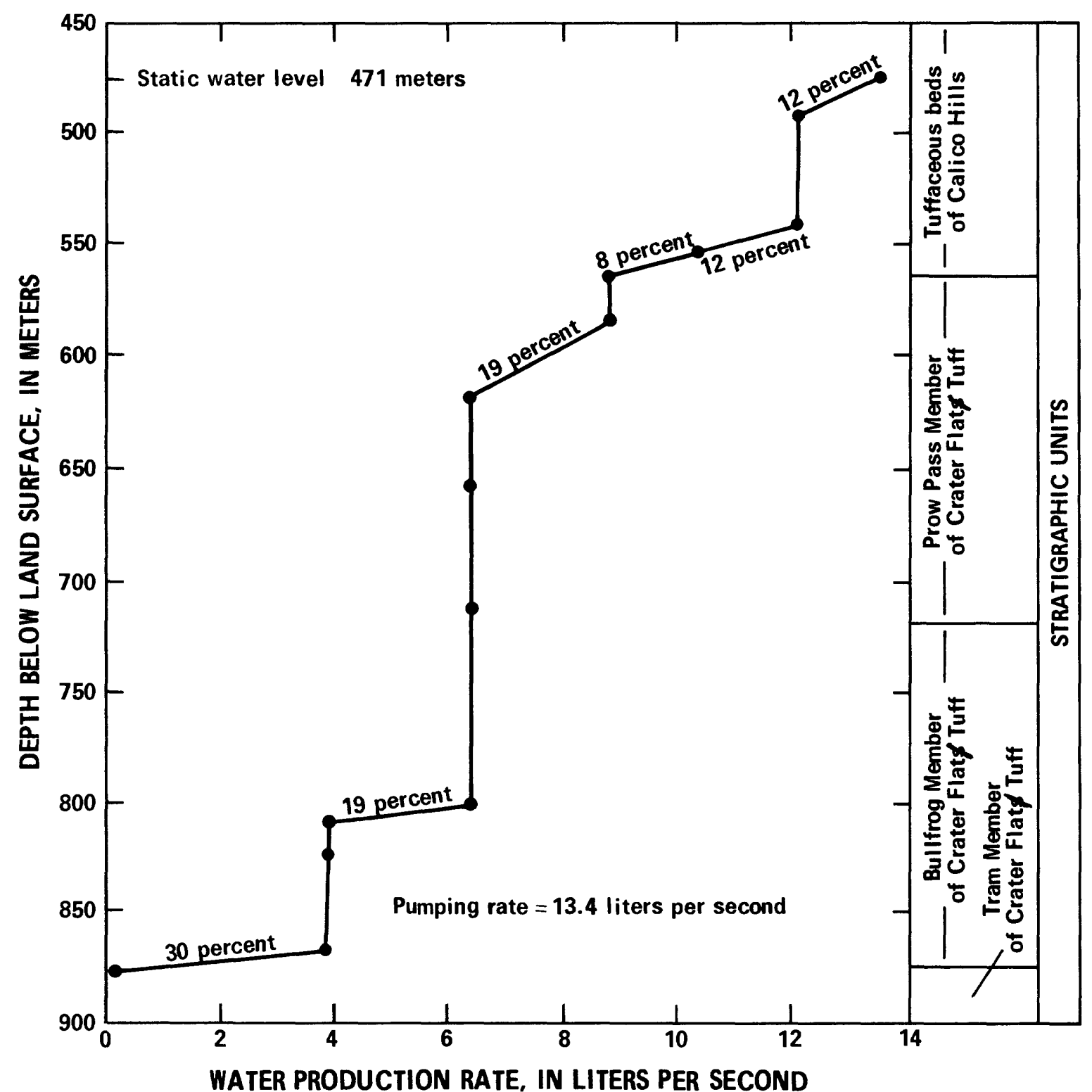

Figure 8.--Borehole-flow survey in test well UE-25b\#1, showing percent of pumping rate produced by intervals. 


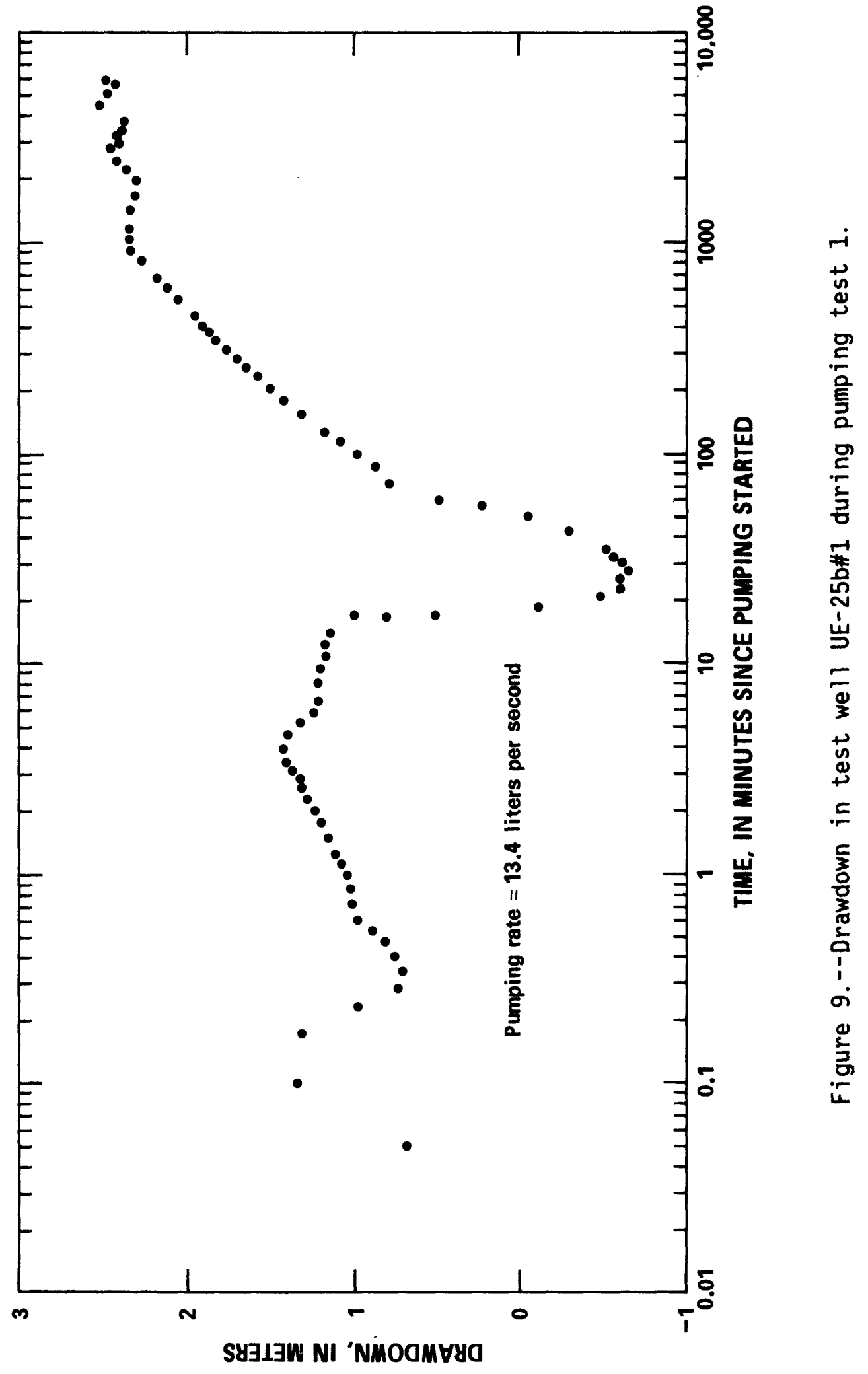




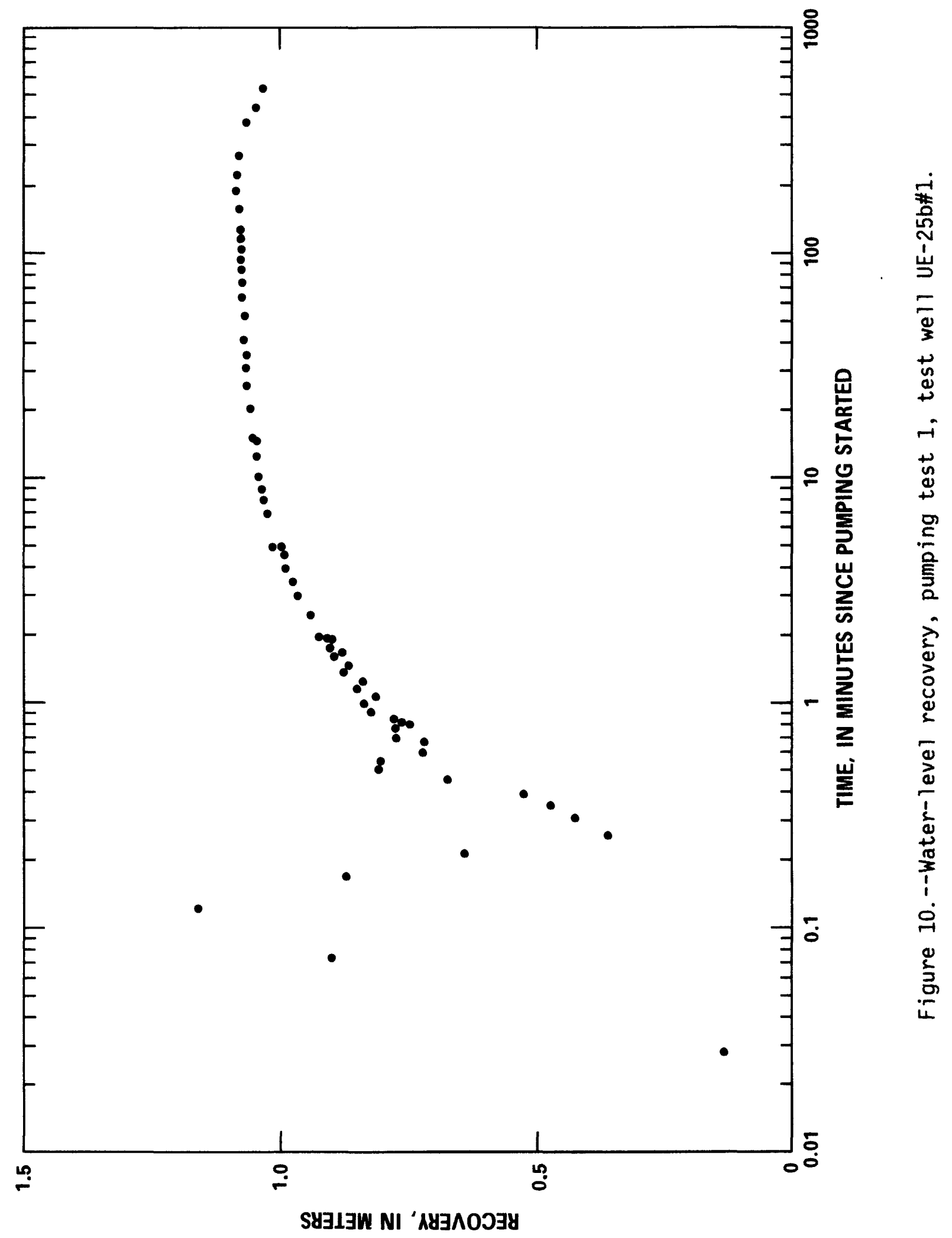




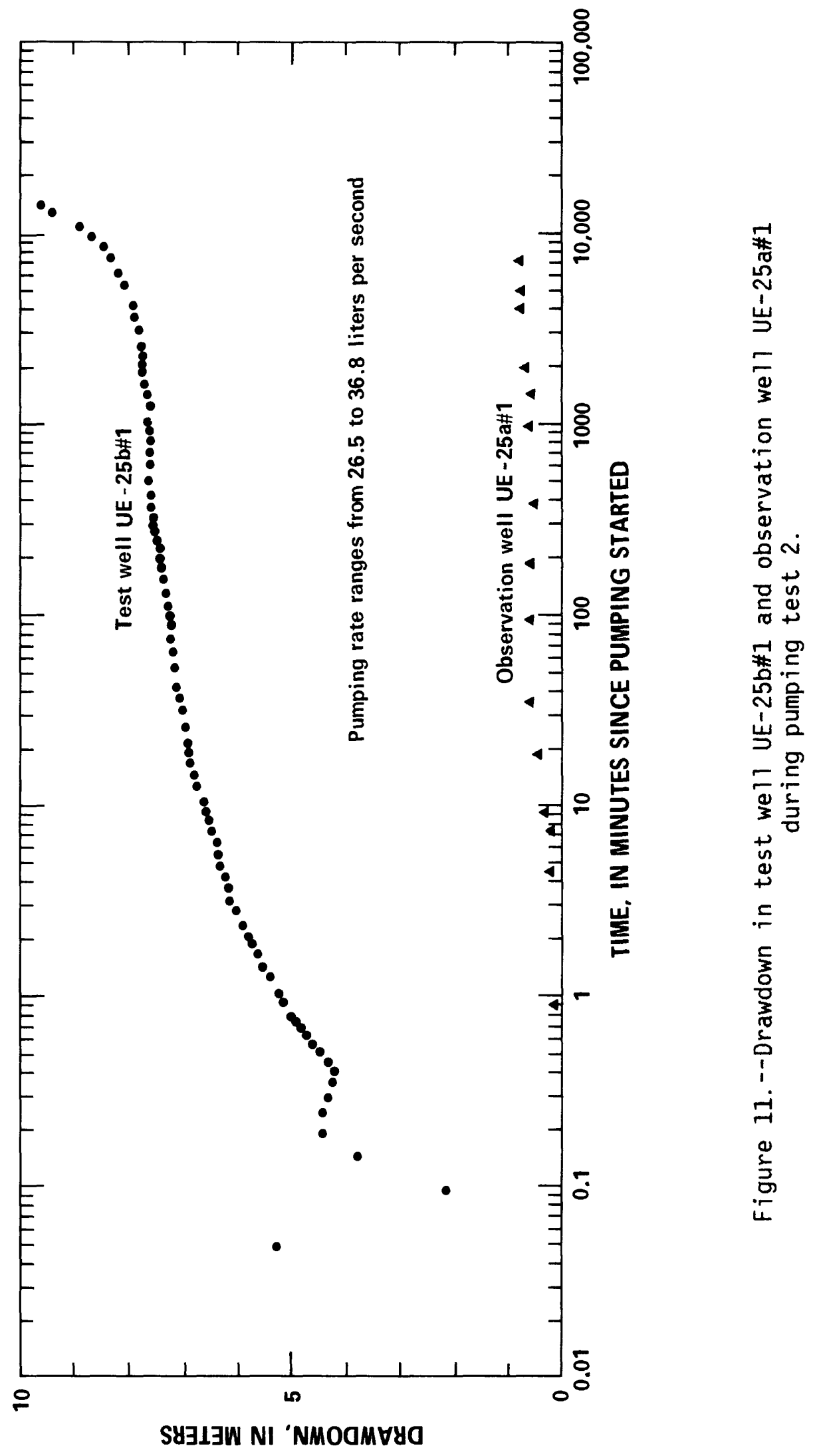




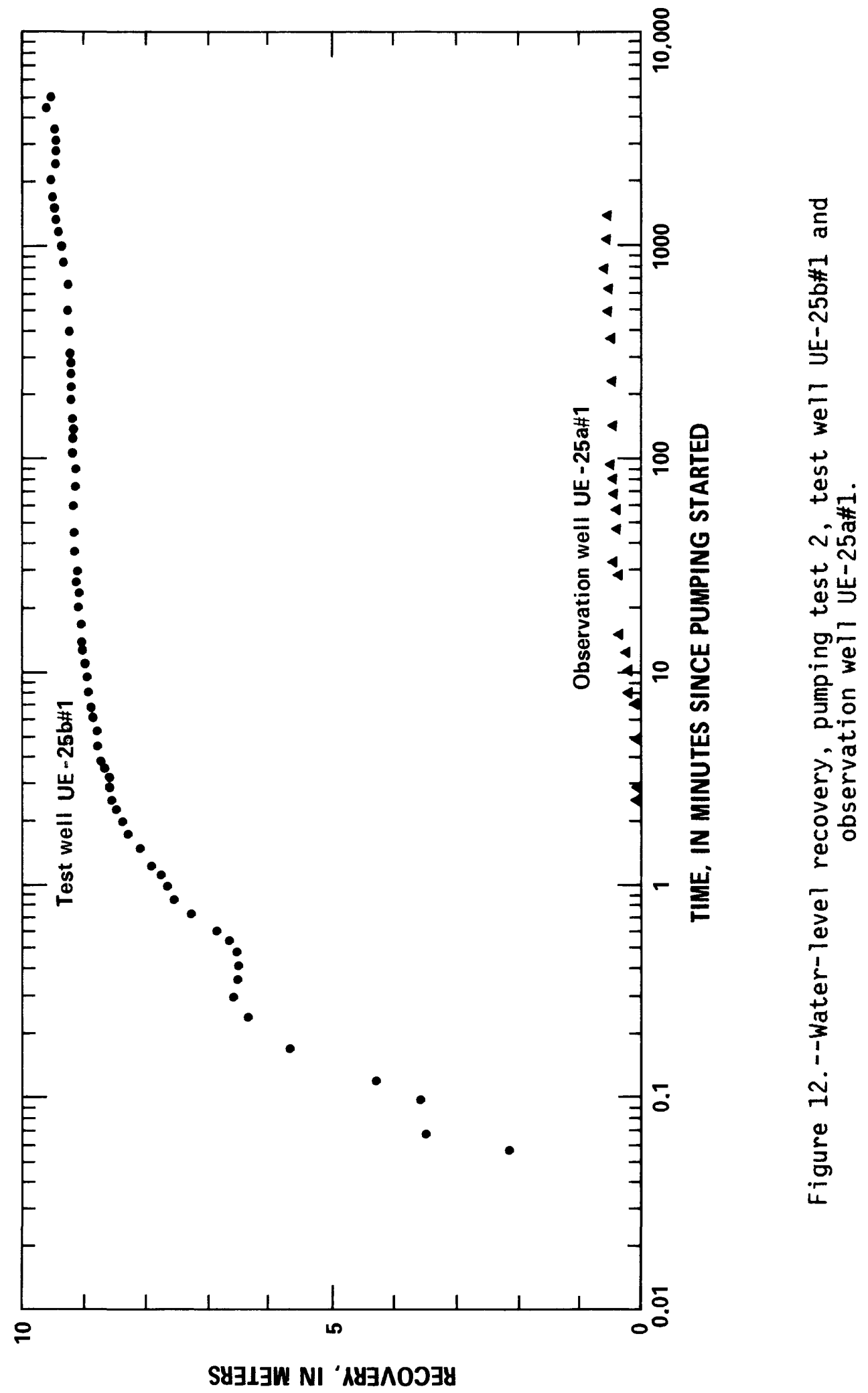




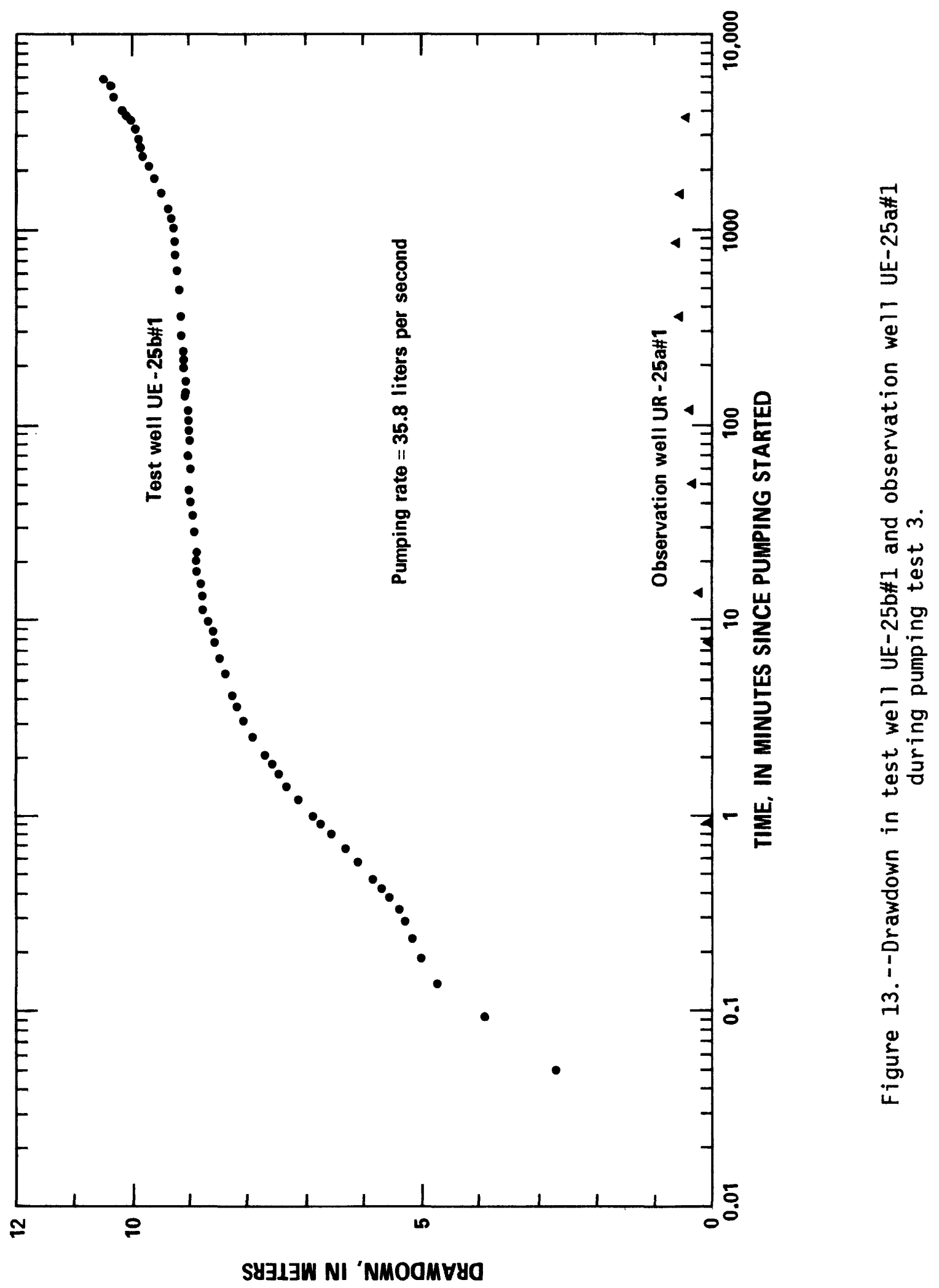




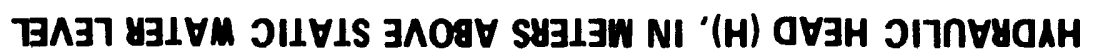

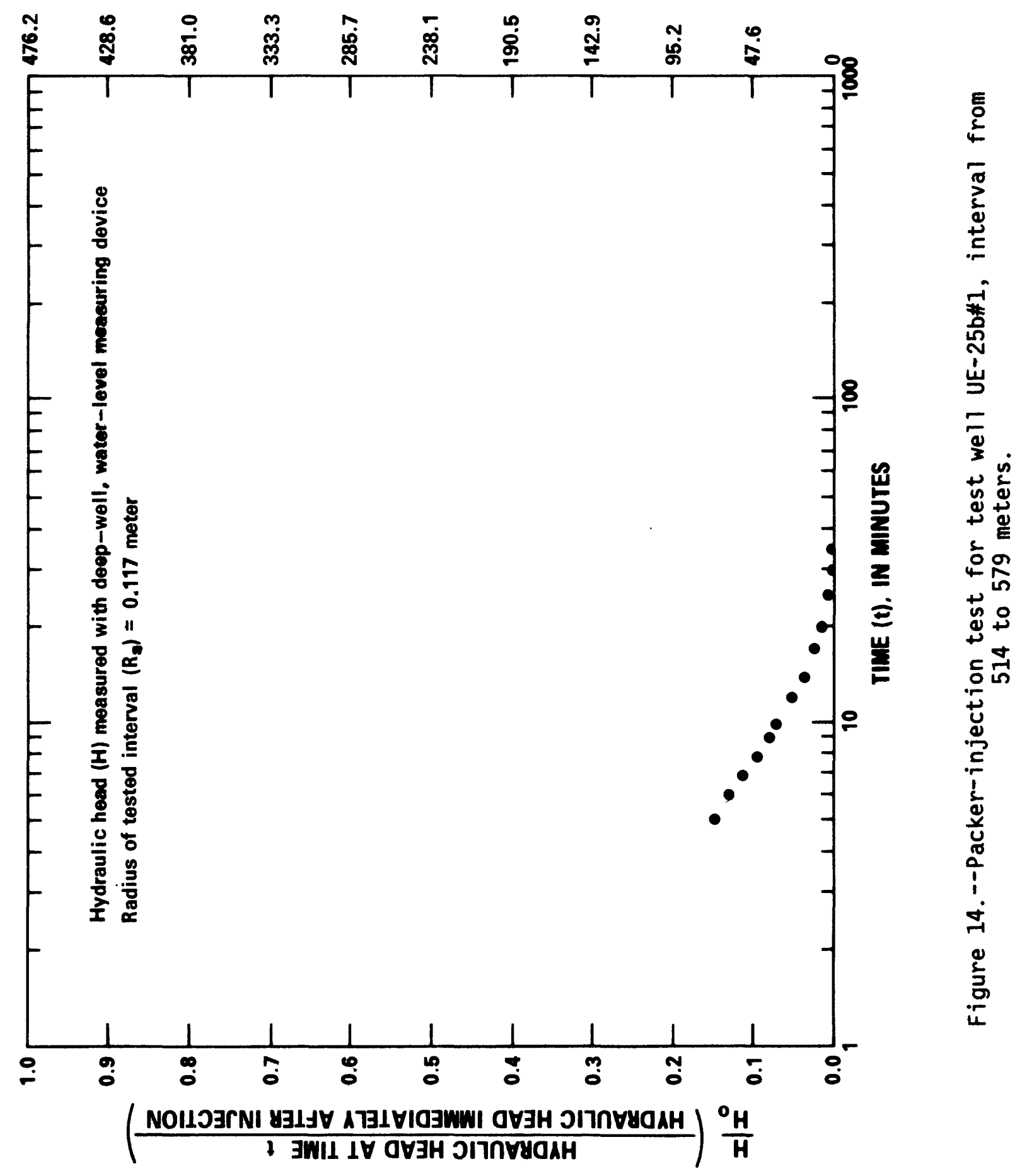




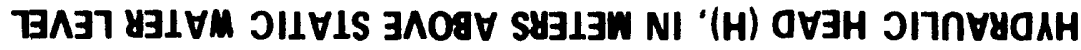

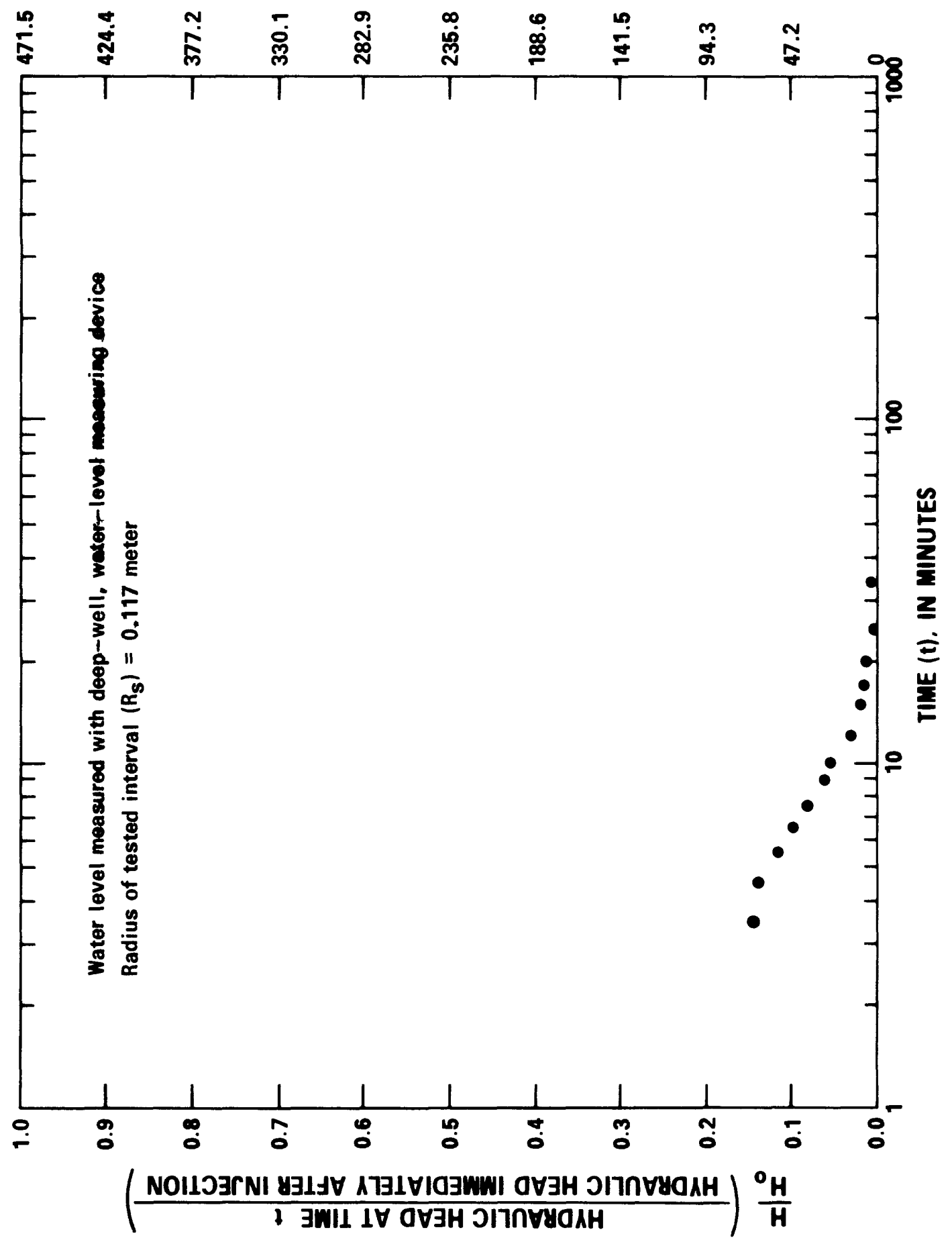

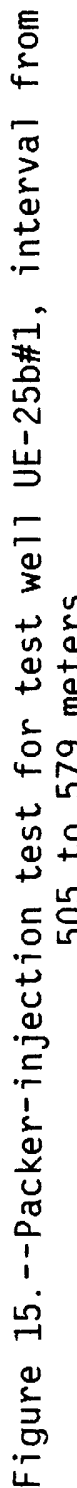




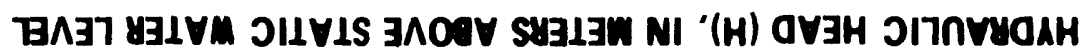

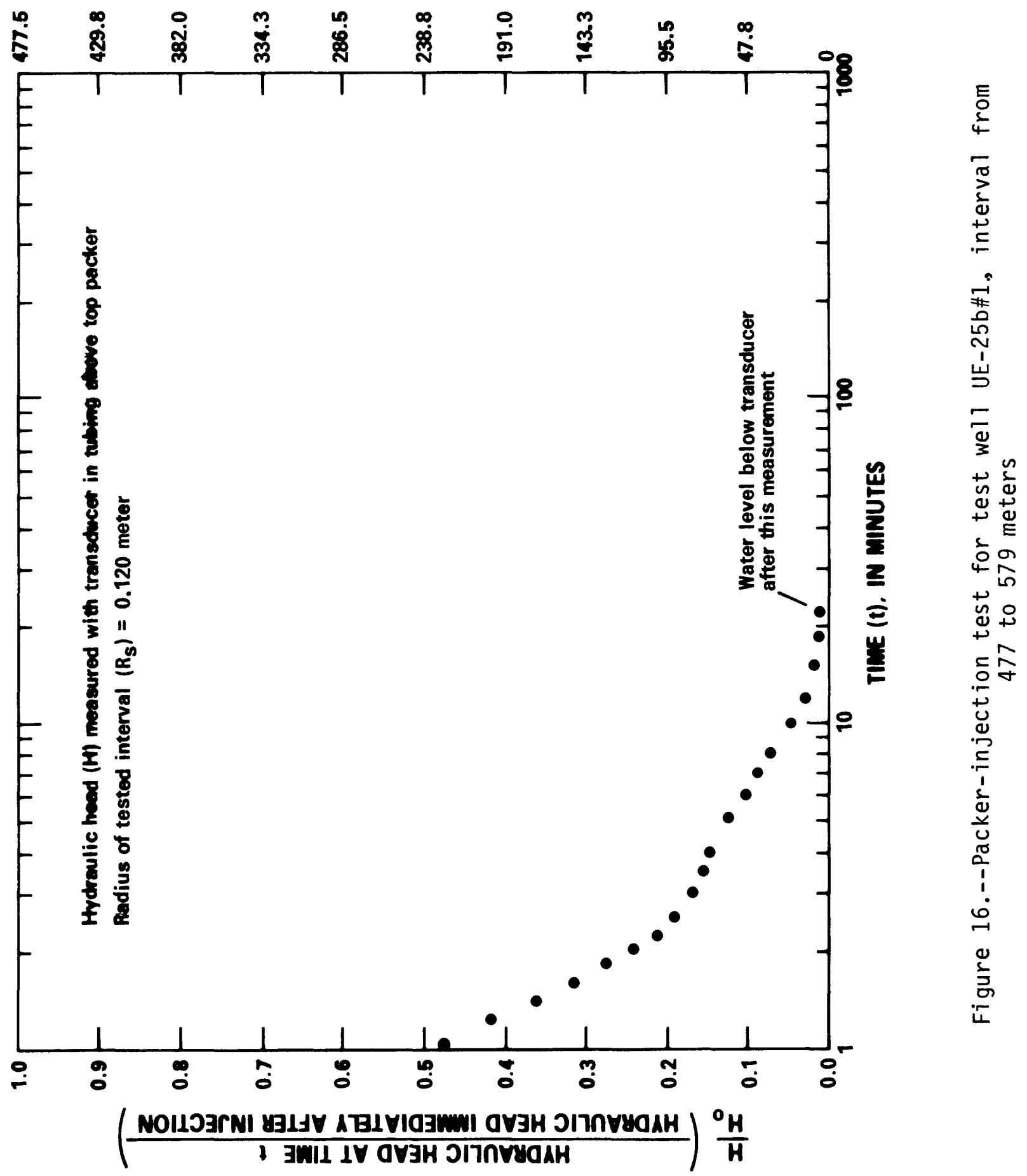




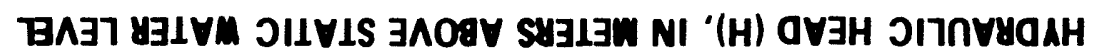

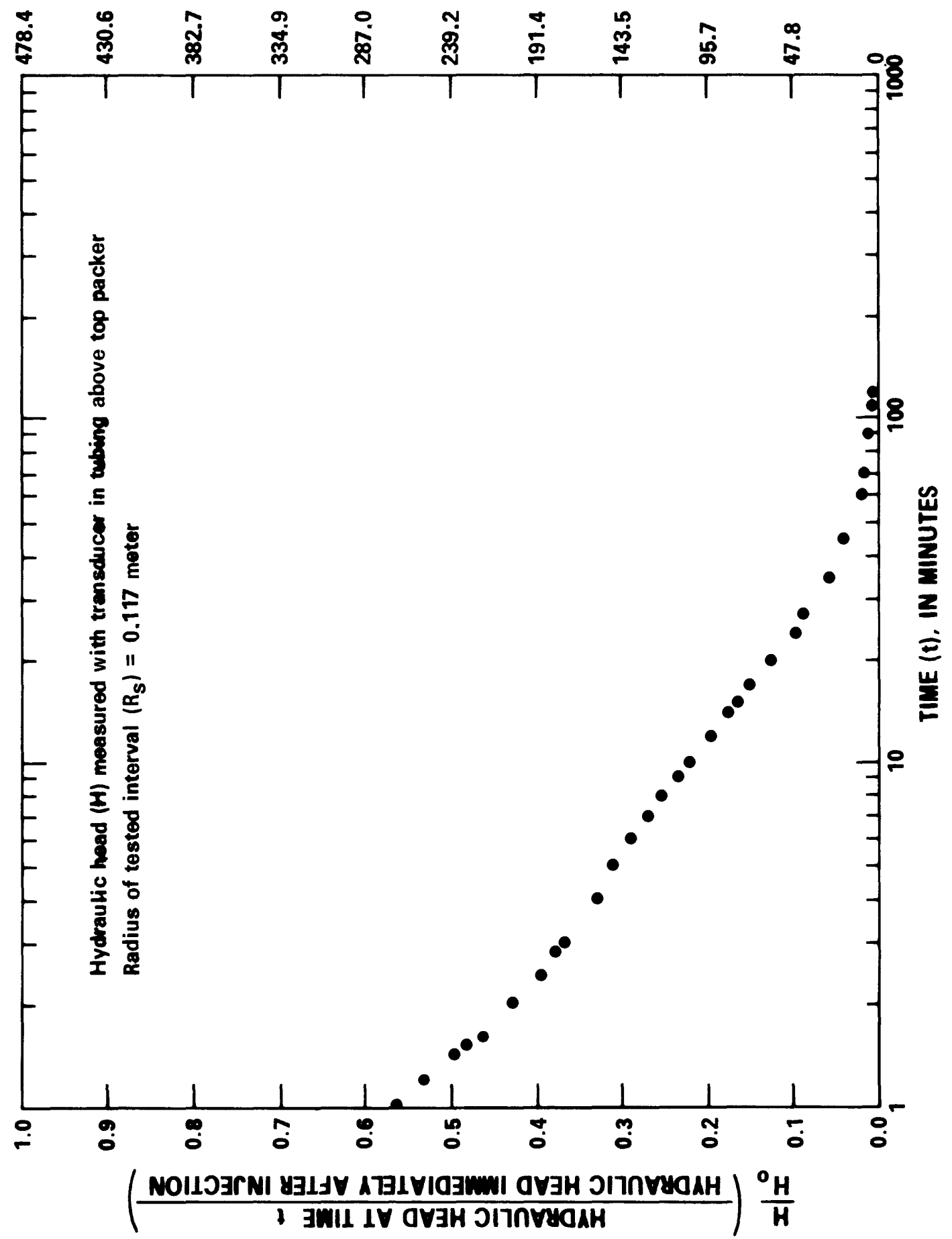

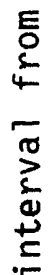

落

岁

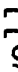

$\frac{\Phi}{3}$

$+\frac{1}{4}$

(1)

$\Phi$

눈

is

용ㅇ

등

苋

.

$\frac{1}{d}$

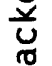

$\frac{0}{1}$

N

奈 


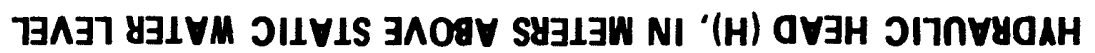

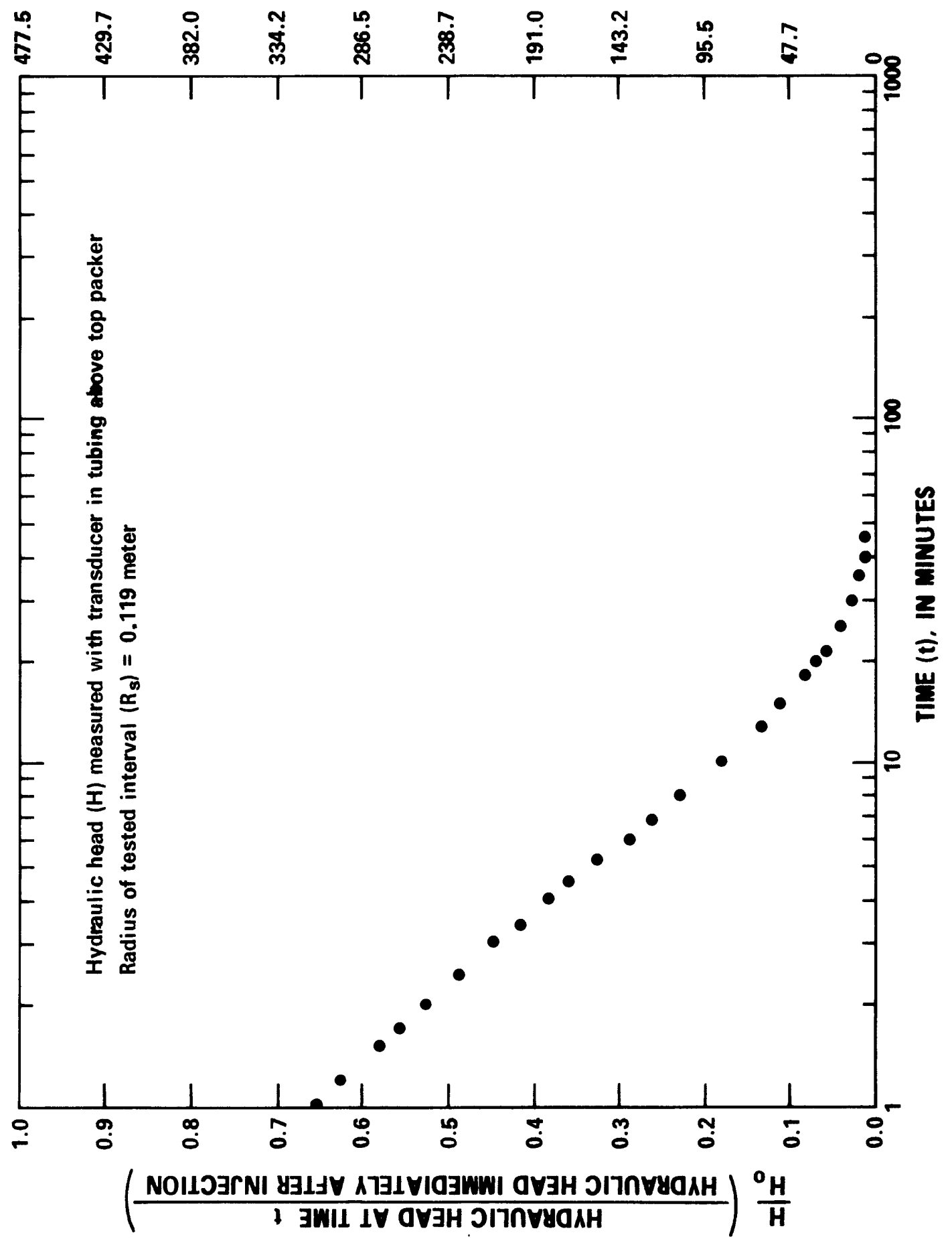

ֻ

䓠

岁

$\bar{\sigma}$

3

$+\frac{n}{a}$

\&

$+\underset{E}{E}$

ธํำ

\&

范

¿

웡

$\frac{2}{2}$

过

E

d

뭉

$\frac{1}{1}$

$\infty$

紊 


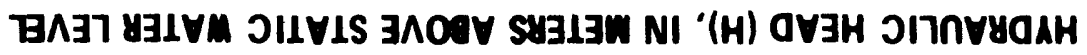

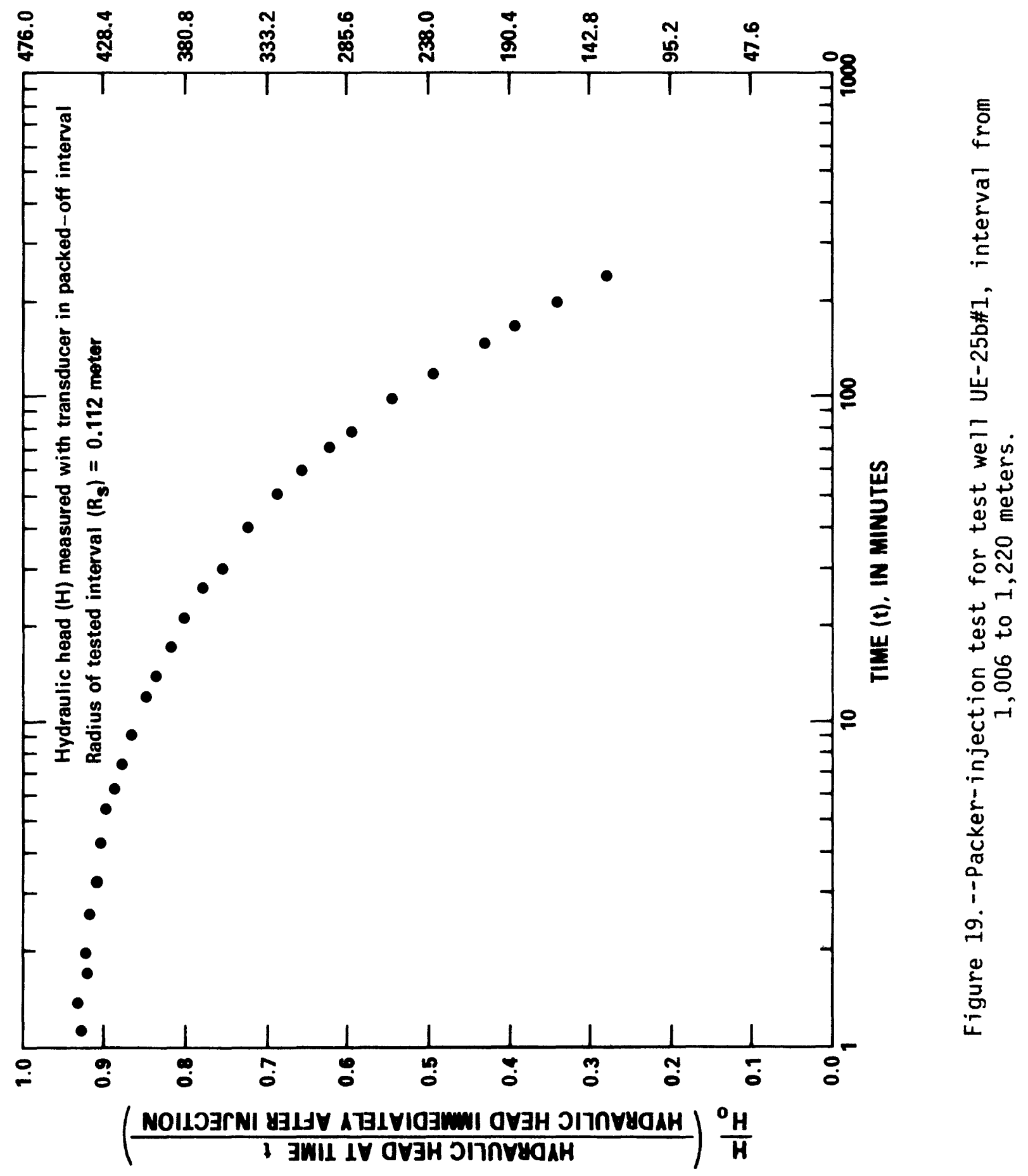




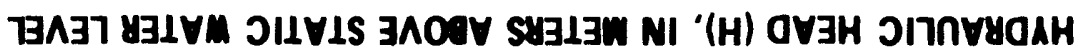

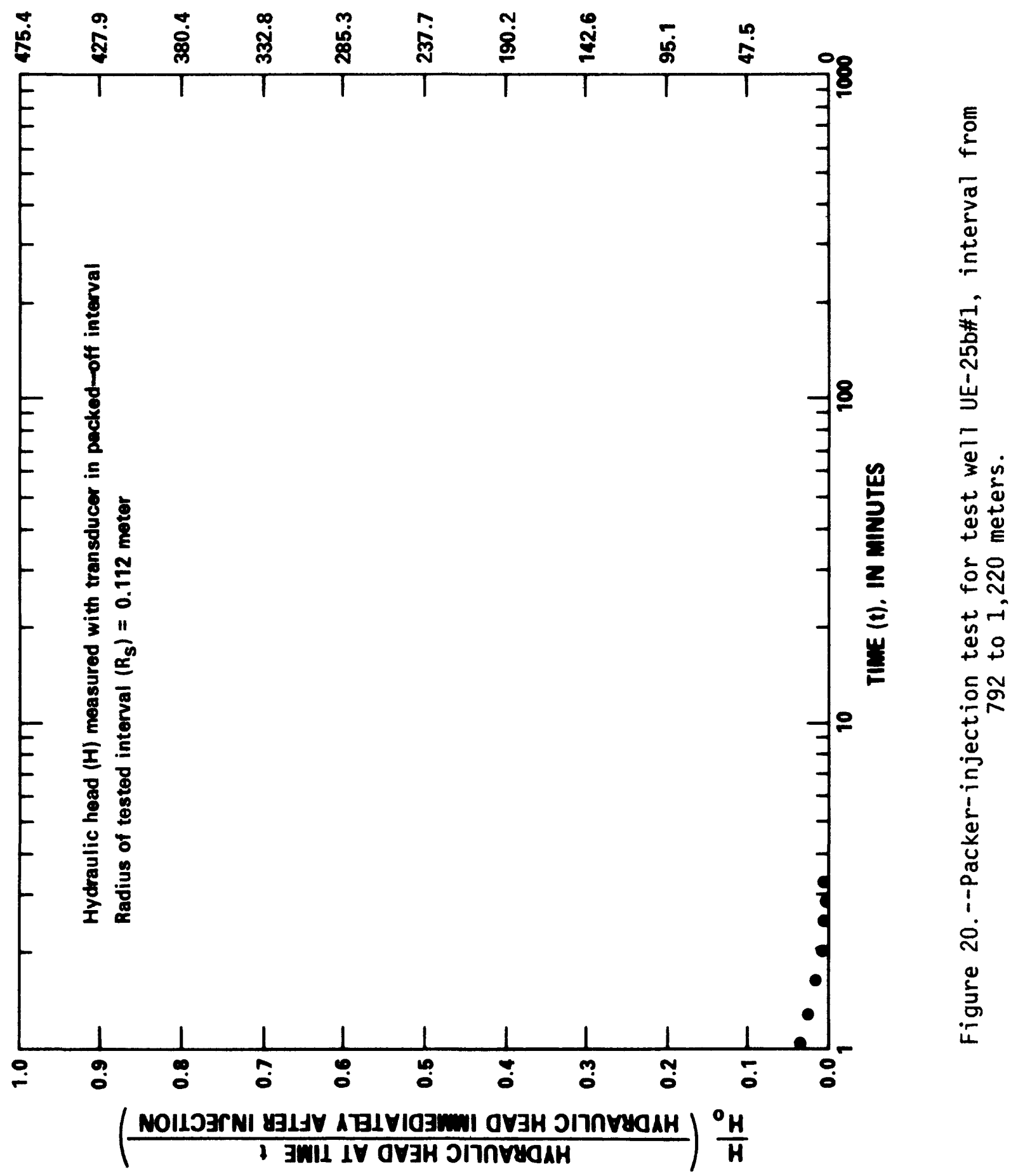




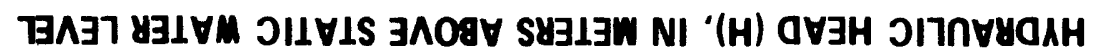

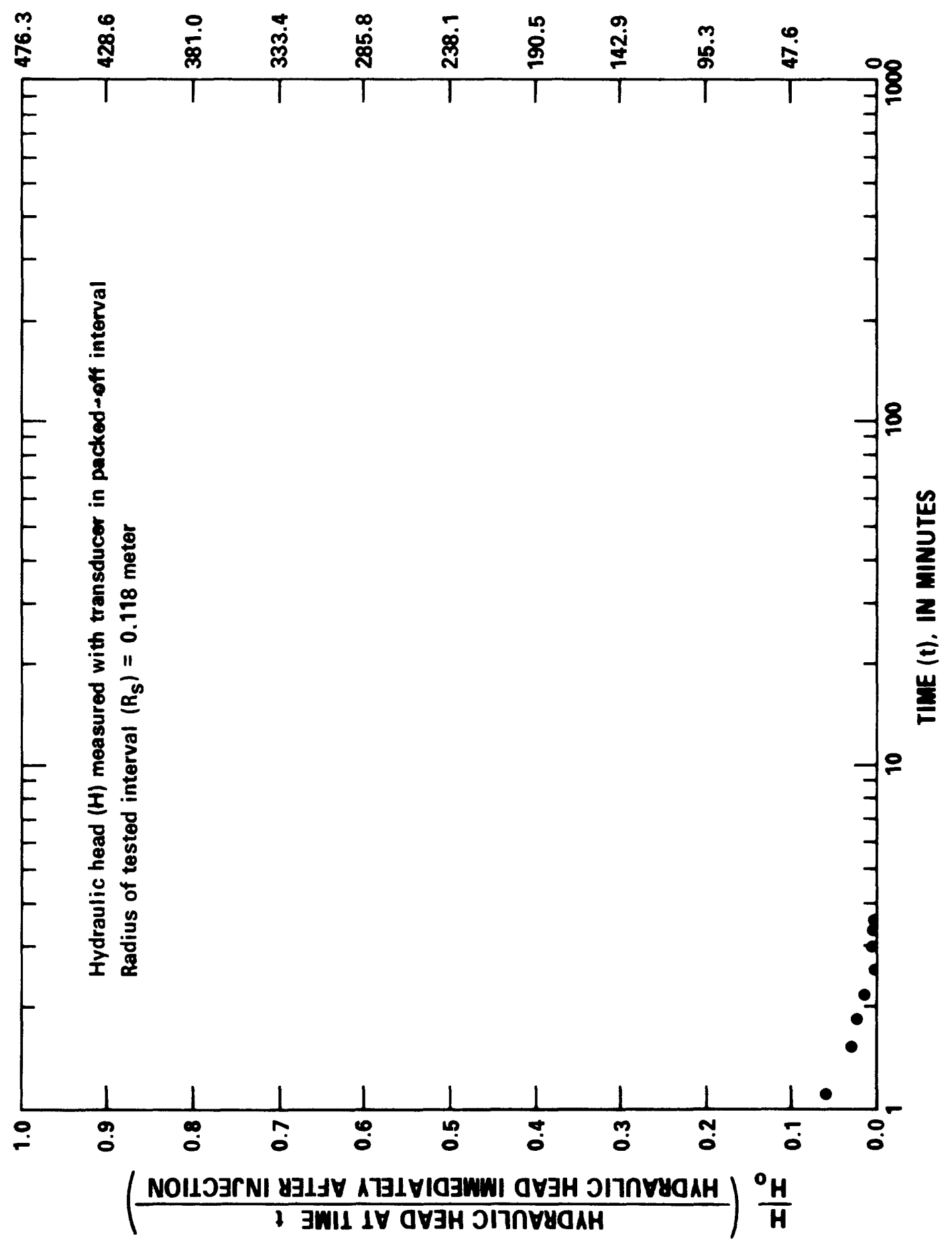

E

䓠

告

$\overline{0}$

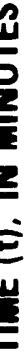

3

is

$\stackrel{\varpi}{\Downarrow}$

ธิ์

$\leftarrow$ \%

峁

동

ᄃㄴㅇㅇ

ư

.

.

过

U

!

$\dot{\sim}$

文 


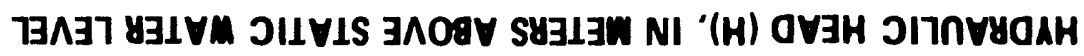

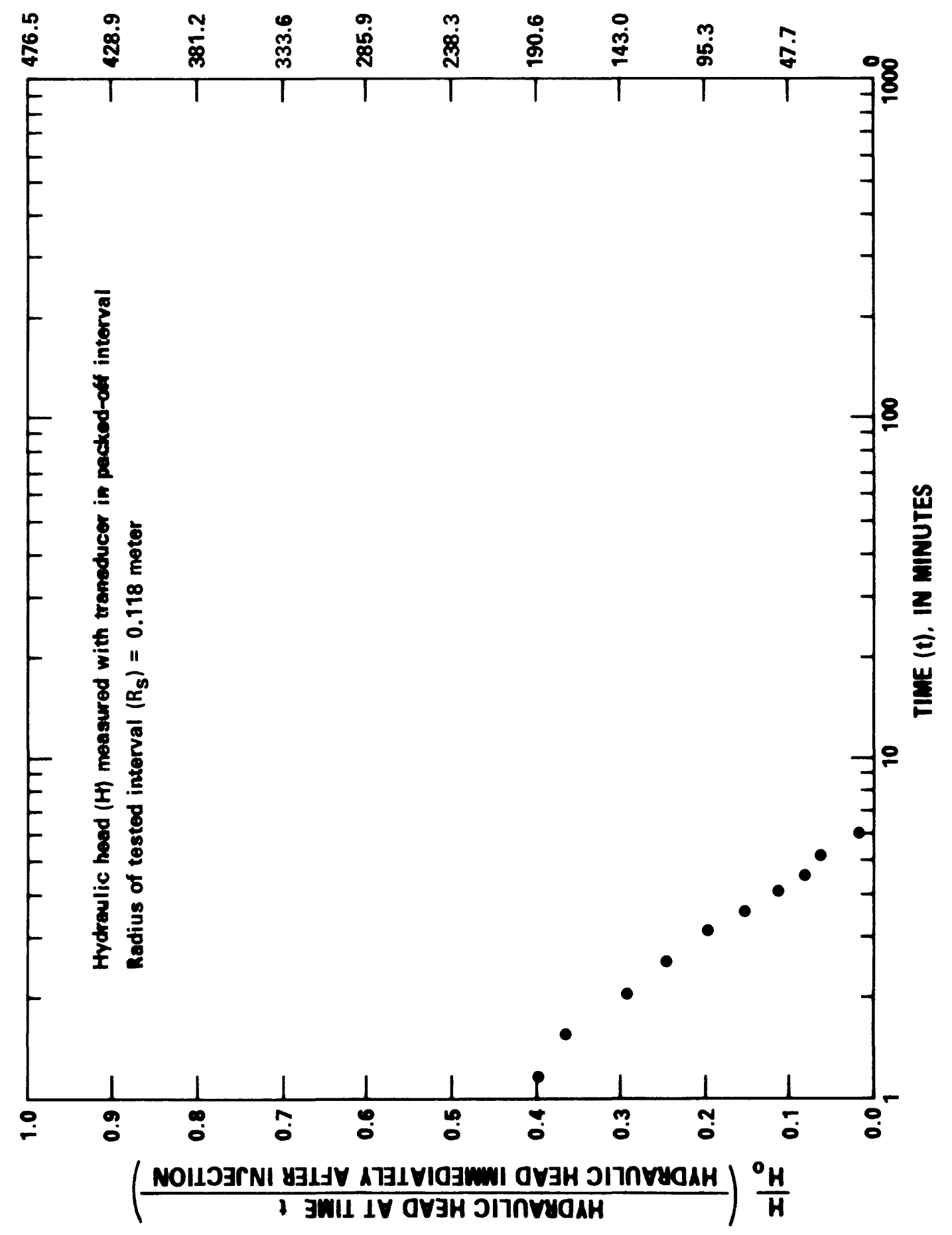

$\approx$

空 


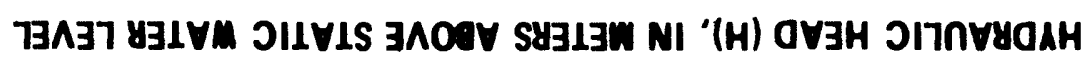

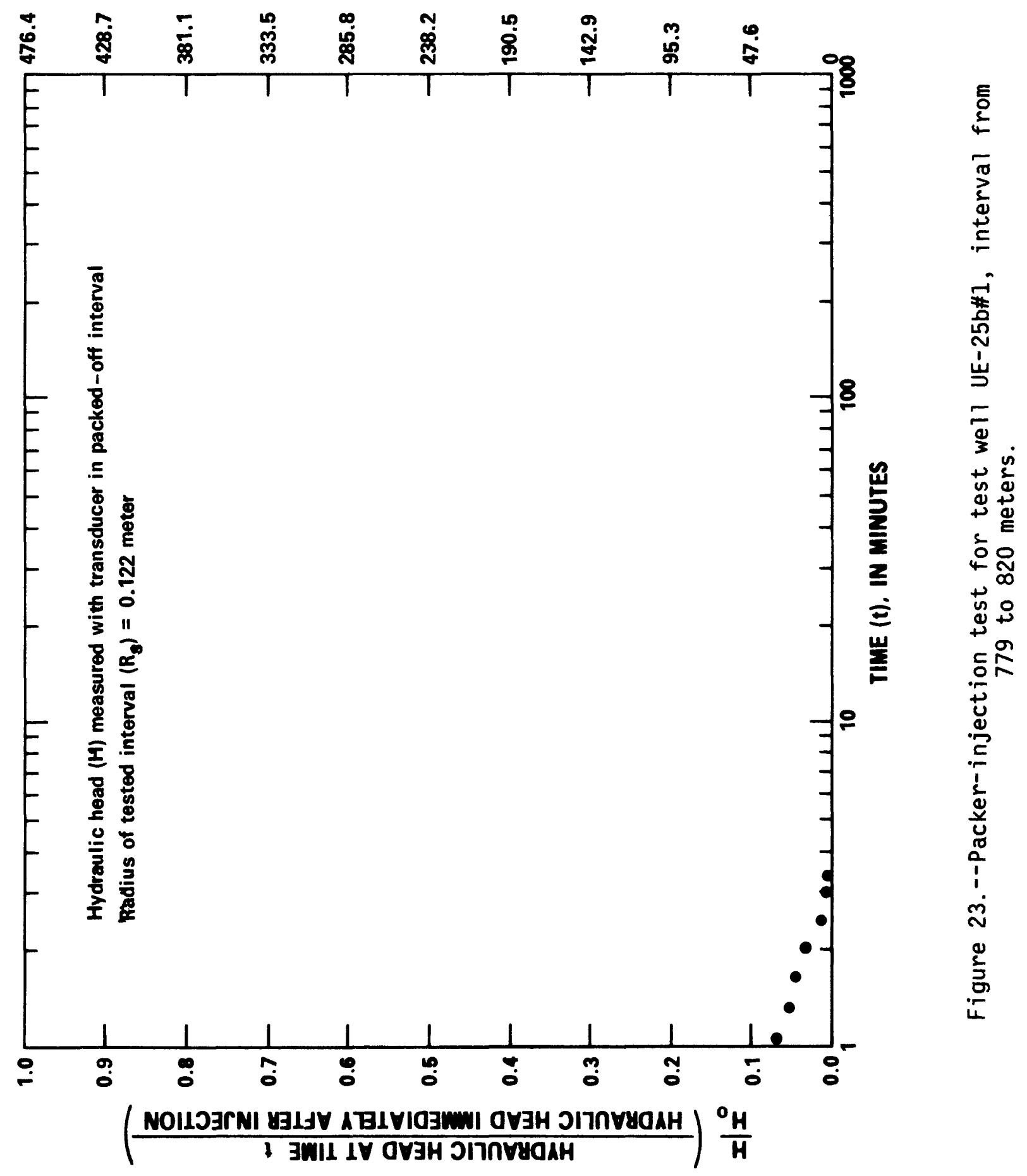




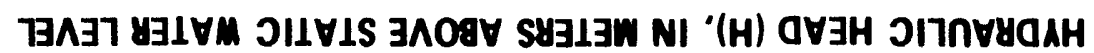

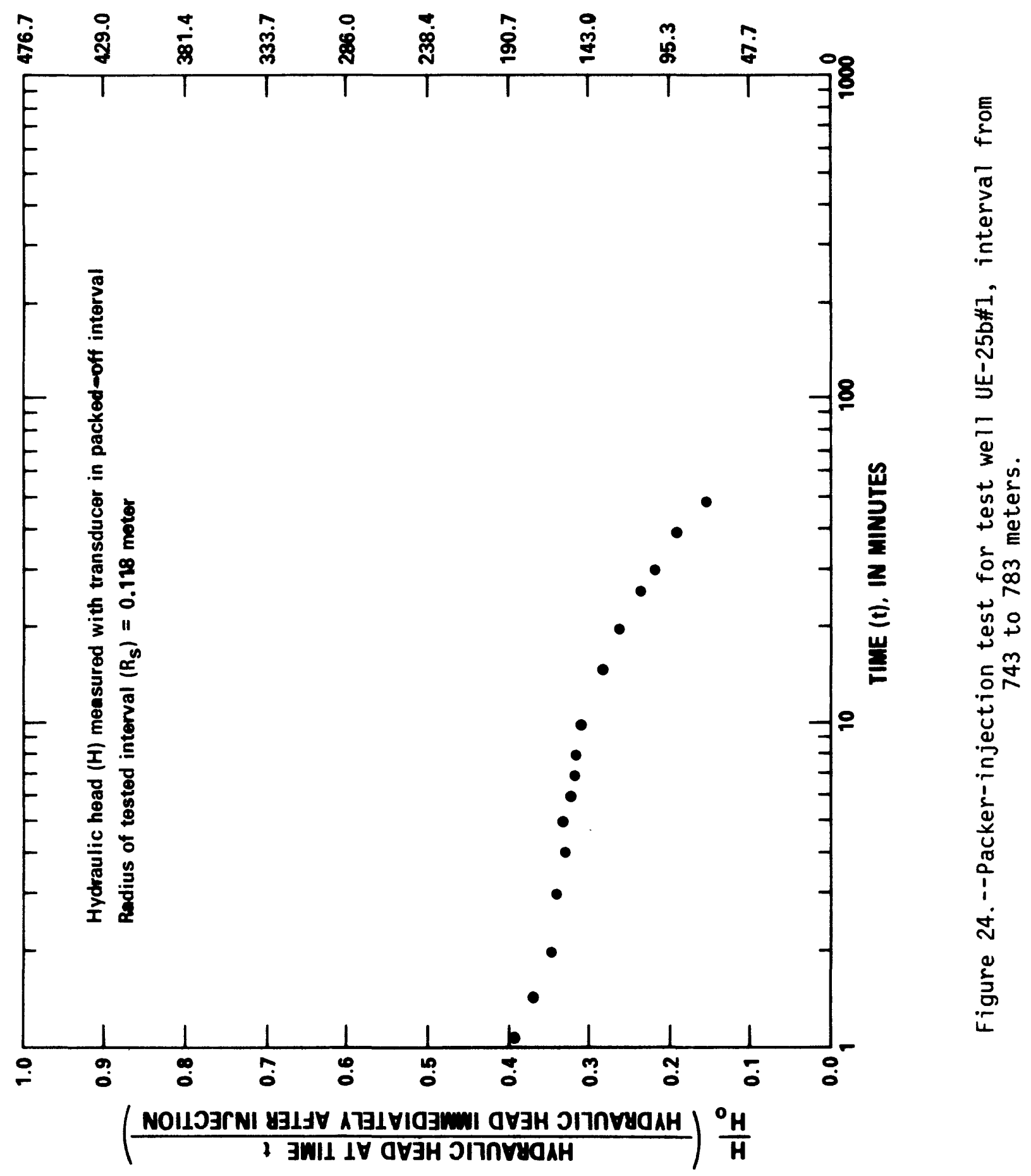




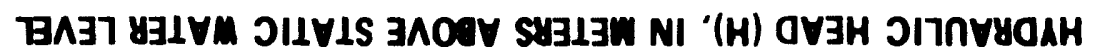

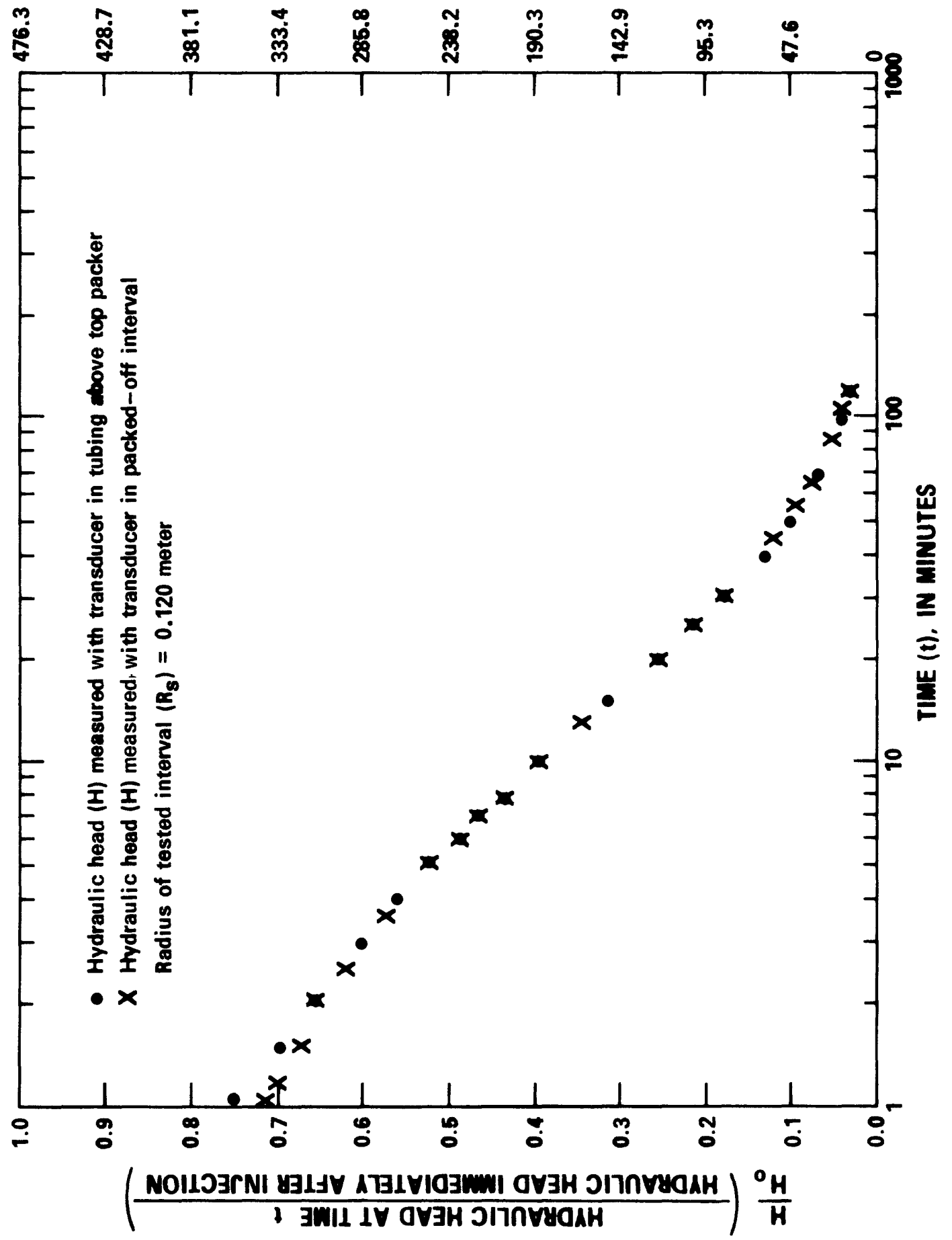




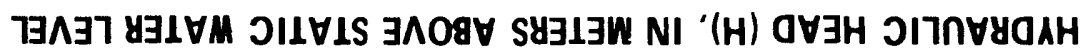

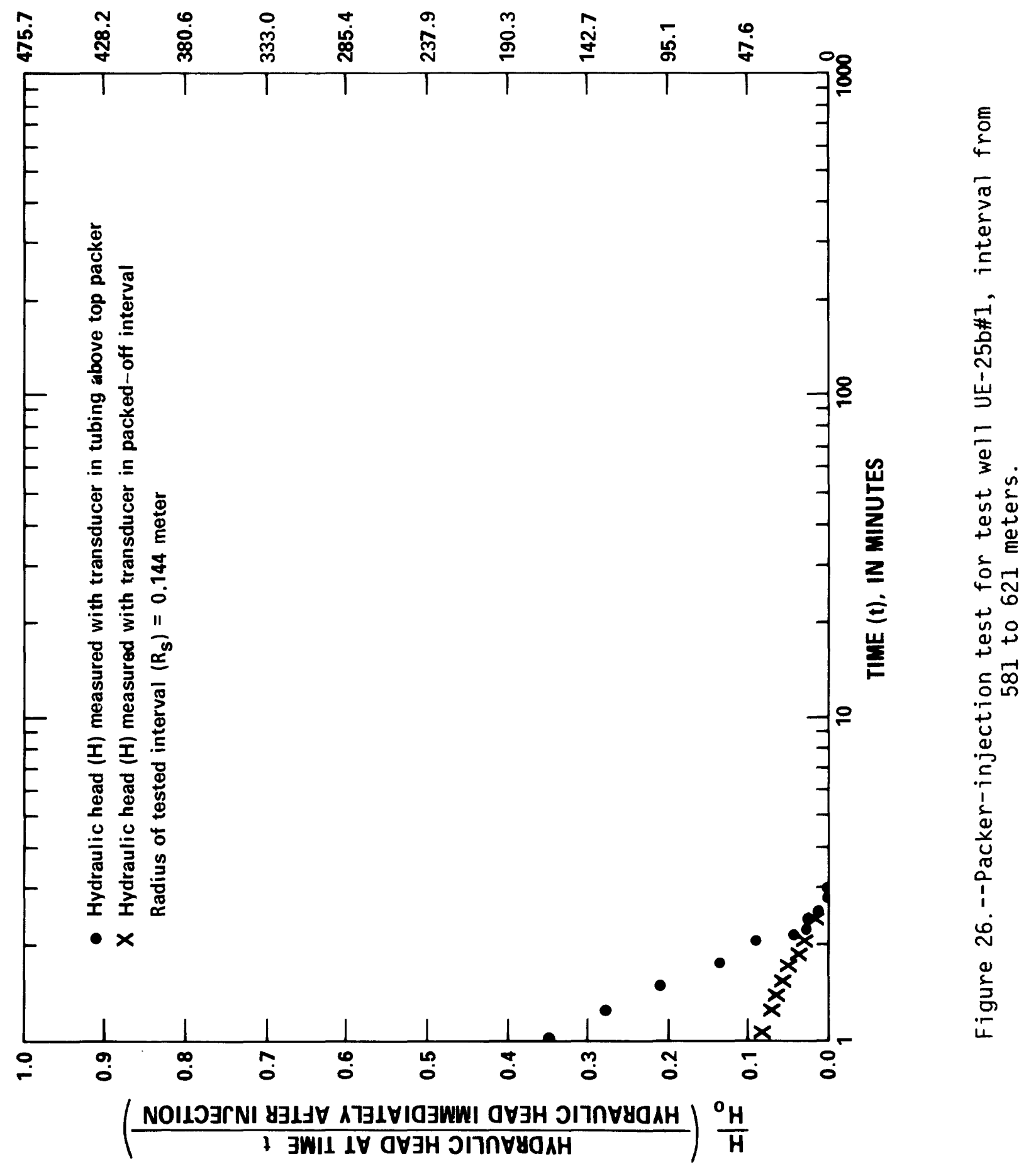




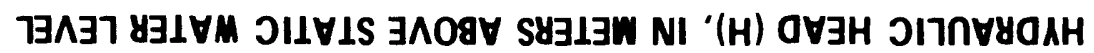

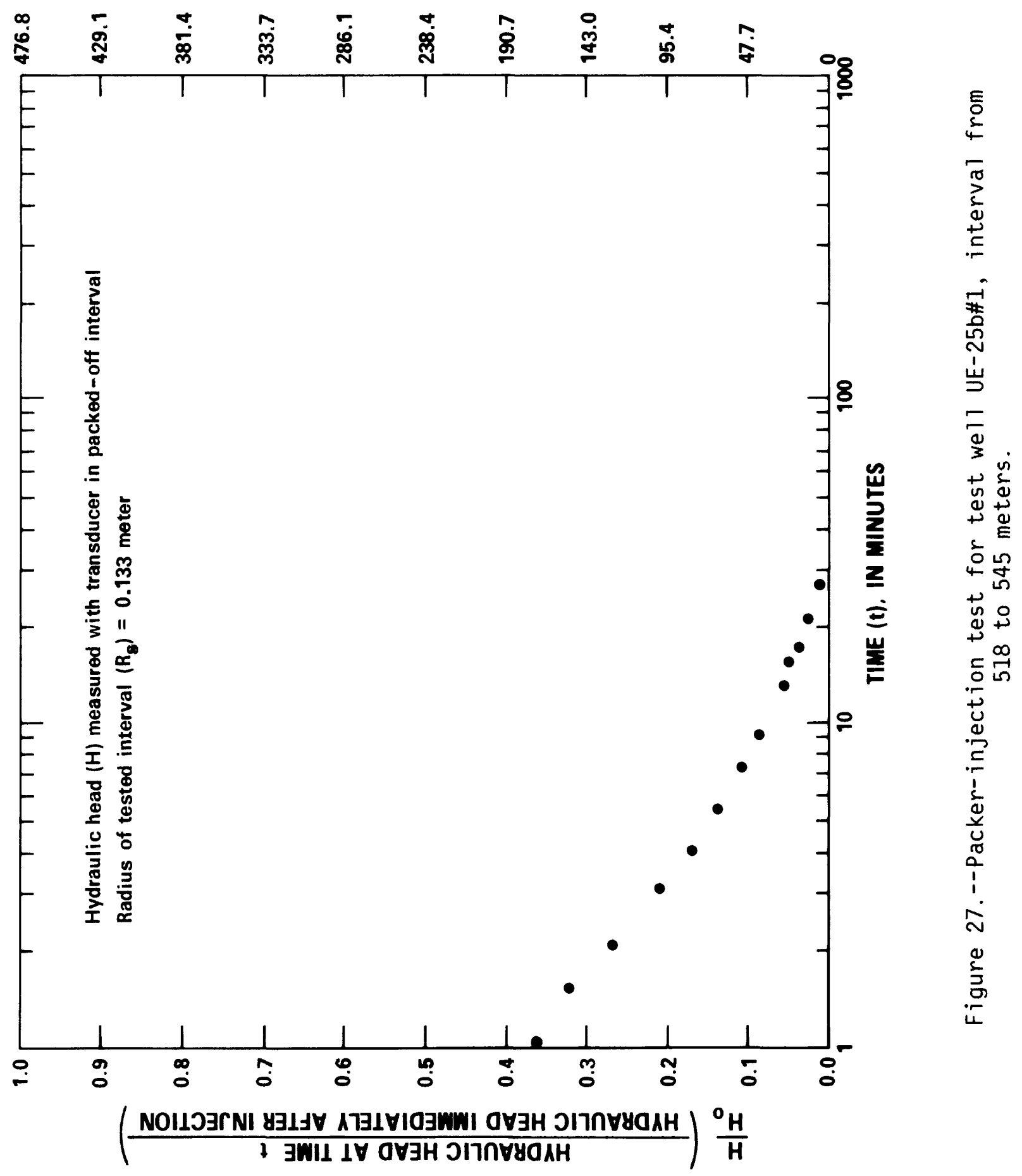




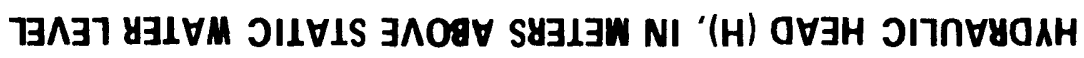

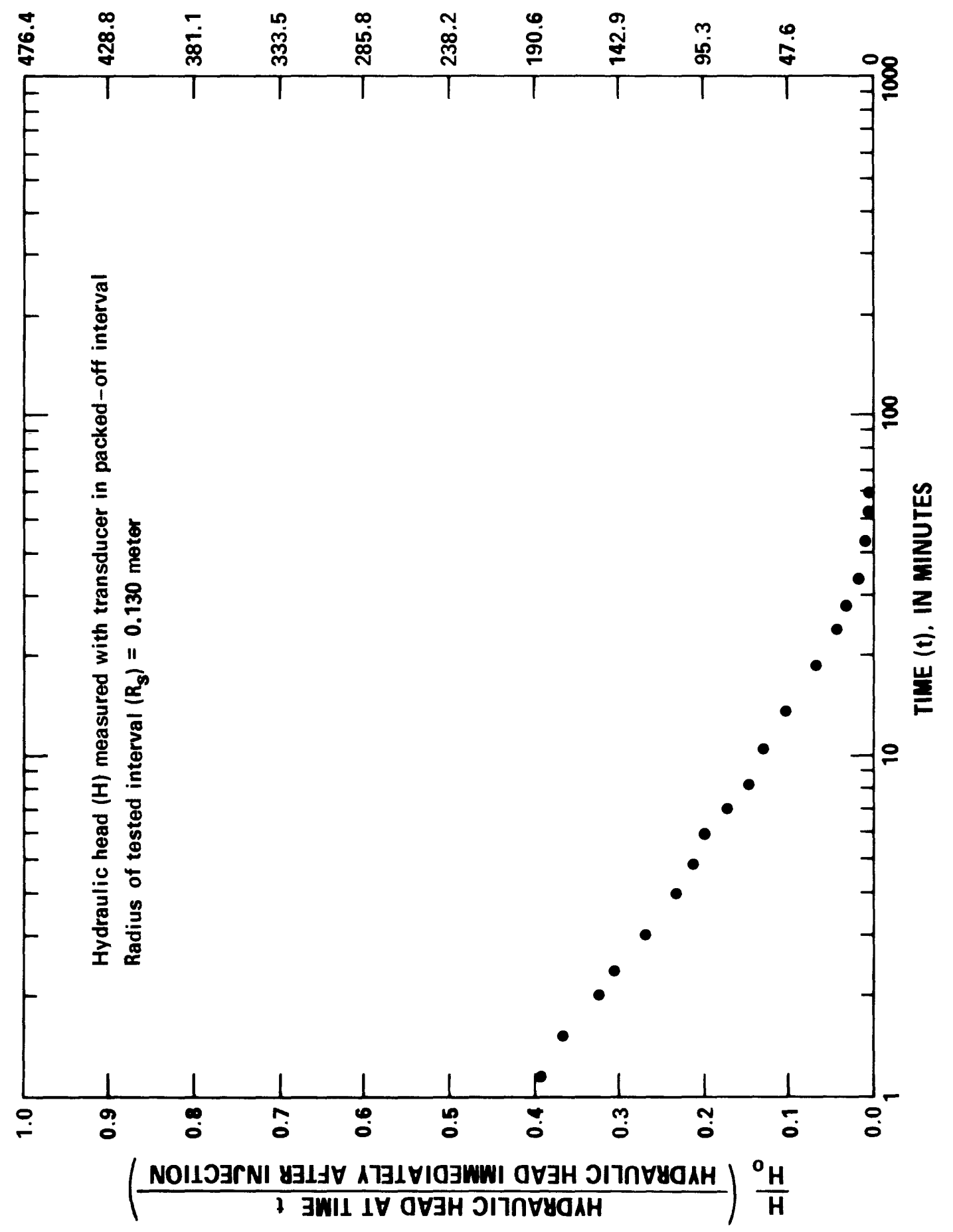

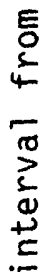

萿

$\stackrel{1}{\sim}$

岁

$\bar{\sigma}$

$\stackrel{\infty}{3}$

is $\frac{n}{d}$

$\stackrel{4}{\sim}$

ธ

46

i

언

ᄃ

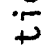

过

它

5

产

$\ddot{U}$

!

$\stackrel{\infty}{\sim}$

告 


\section{Chemical Analyses of Water}

A composite ground-water sample from the tuffaceous rocks of the wateryielding interval penetrated by the well was collected and analyzed for chemical constituents and other properties (table 5). The sample was collected at the end of the third pumping test.

A lithium chloride spike was put into water added to the well during drilling, coring, and injection tests in order to provide an indication of the completeness of clean-out prior to water sampling. The well was pumped intensively before sampling to provide more representative samples. 
Table 5.--Results of chemical analyses of water samples from test we11 UE-25b\#1 1

[A11 units are milligrams per liter unless otherwise indicated]

\begin{tabular}{|c|c|}
\hline $\begin{array}{l}\text { Chemical constituents or } \\
\text { physical properties }\end{array}$ & $\begin{array}{l}\text { Sampled during pumping of depth } \\
\text { interval from } 471 \text { to } 1,220 \text { meters }\end{array}$ \\
\hline $\begin{array}{l}\text { Bicarbonate-field }\left(\mathrm{HCO}_{3}\right) \\
\text { Calcium (Ca) } \\
\text { Chloride (Cl) } \\
\text { Fluoride (F) } \\
\text { Lithium (Li) }\end{array}$ & $\begin{array}{c}139 \\
17 \\
8.5 \\
1.6 \\
.22\end{array}$ \\
\hline $\begin{array}{l}\text { Magnesium }(\mathrm{Mg}) \\
\text { Potassium }(\mathrm{K}) \\
\text { Silica }\left(\mathrm{SiO}_{2}\right) \\
\text { Strontium }(\mathrm{Sr}) \\
\text { Sulfate }\left(\mathrm{SO}_{4}\right)\end{array}$ & $\begin{array}{l}.59 \\
3.5 \\
52.038 \\
22^{.5}\end{array}$ \\
\hline $\begin{array}{l}\text { Tritium }\left({ }^{3} \mathrm{H}\right) \\
\text { Oxygen-18/oxygen-16 }\left(\partial^{18} \mathrm{O}\right) \\
\text { Deuterium-hydrogen }\left(\partial^{2} \mathrm{H}\right) \\
\text { Carbon-13/carbon-12 }\left(\partial^{13} \mathrm{C}\right) \\
\text { Dissolved solids (sum) }\end{array}$ & $\begin{array}{c}<200 \text { picocuries/liter } \\
2-13.4 \\
3-101 \\
4-10.4 \\
218\end{array}$ \\
\hline $\begin{array}{l}\text { Dissolved solids (residue at } 180^{\circ} \text { Celsius) } \\
\text { Hardness (as milligrams per liter } \mathrm{CaCO}_{3} \text { ) } \\
\text { Temperature (degrees Celsius) } \\
\text { Specific conductance } \\
\text { pH (field) } \\
\text { Age (carbon-14 dating) }\end{array}$ & $\begin{array}{l}225 \\
45 \\
36 \\
5281 \text { microsiemens } \\
7.3 \text { units } \\
14,100 \text { years }\end{array}$ \\
\hline
\end{tabular}
Colorado.

${ }^{1}$ Chemical analyses made by U.S. Geological Survey laboratory, Denver,

2Deviation of oxygen-18/oxygen-16 ratio of sample from standard mean ocean water (SMOW) relative to SMOW, in parts per thousand.

${ }^{3}$ Deviation of deuterium/hydrogen ratio of sample from SMOW relative to SMOW, in parts per thousand.

${ }^{4}$ Deviation of carbon-13/carbon-12 ratio of sample from PeeDee Belemnite Standard (PDB) relative to PDB, in parts per thousand.

${ }^{5}$ Equivalent to micromhos per centimeter at $25^{\circ}$ Celsius. 


\section{SELECTED REFERENCES}

Ferris, J. G., Knowles, D. B., Brown, R. H., and Stallman, R. W., 1962, Theory of aquifer tests: U.S. Geological Survey Water-Supply Paper 1536-E, $174 \mathrm{p}$.

Lohman, S. W., 1972, Ground-water hydraulics: U.S. Geological Survey Professional Paper 708, $70 \mathrm{p}$.

Papadopulos, S. S., Bredehoeft, J. D., and Cooper, H. H., 1973, The analys is of slug test data: Water Resources Research, v. 9, no. 4, p. 1087-1089.

Schlumberger Limited, 1972, Log interpretation, Volume 1--Principles: New York, $113 \mathrm{p}$. 